\title{
Min-max model predictive control of nonlinear systems: A unifying overview on stability
}

\author{
D. M. Raimondo ${ }^{1, *}$, D. Limon ${ }^{2, * *}$, M. $\operatorname{Lazar}^{3, * * *}$ L. Magni $^{1}$ and E. F. Camacho ${ }^{2, * * * *}$ \\ ${ }^{1}$ Dipartimento di Informatica e Sistemistica, Università di Pavia, via Ferrata 1, 27100 Pavia, Italy; \\ 2 Departamento de Ingeniería de Sistemas y Automática, Universidad de Sevilla, \\ Escuela Superior de Ingenieros, Camino de los Descubrimientos s/n 41092 Sevilla, Spain; \\ 3 Department of Electrical Engineering, Eindhoven University of Technology, \\ P.O. Box 513, 5600 MB Eindhoven, The Netherlands
}

\begin{abstract}
Min-Max model predictive control (MPC) is one of the few techniques suitable for robust stabilization of uncertain nonlinear systems subject to constraints. Stability issues as well as robustness have been recently studied and some novel contributions on this topic have appeared in the literature. In this survey, we distill from an extensive literature a general framework for synthesizing min-max MPC schemes with an a priori robust stability guarantee. Firstly, we introduce a general prediction model that covers a wide class of uncertainties, which includes bounded disturbances as well as state and input dependent disturbances (uncertainties). Secondly, we extend the notion of regional Input-to-State Stability (ISS) in order to fit the considered class of uncertainties. Then, we establish that the standard min-max approach can only guarantee practical stability. We concentrate our attention on two different solutions for solving this problem. The first one is based on a particular design of the stage cost of the performance index, which leads to a $\mathcal{H}_{\infty}$ strategy, while the second one is based on a dual-mode strategy. Under fairly mild assumptions both controllers guarantee Input-to-State Stability of the resulting closed-loop system. Moreover, it is shown that the nonlinear auxiliary control law introduced in [29] to solve the $\mathcal{H}_{\infty}$ problem can be used, for nonlinear systems affine in control, in all the proposed min-max schemes and also in presence of state independent disturbances. A simulation example illustrates the techniques surveyed in this article.
\end{abstract}

Keywords: nonlinear model predictive control, input-to-state stability, robust control

\section{Introduction}

Model Predictive Control (MPC) is a widely used technique for its facility of handling constraints and model uncertainties (see e.g. the books [3, 28, 44] and the survey papers [39, 7]). Several results have been proposed concerning robustness of nominal MPC and robust MPC design (see e.g. [35, 33] for a survey, the reference therein, and some of the most recent papers $[12,13,36,6,26,43])$. A first method for the design of robust MPC consists in minimizing a nominal performance index while imposing the fulfillment of constraints for each admissible disturbance, see [24, 41]. This calls for the inclusion in the problem formulation of tighter state and terminal constraints and leads to very conservative solutions or even to unfeasible problems. With

Correspondence to: L. Magni E-mail: lalo.magni@unipv.it.

${ }^{*}$ E-mail: davide.raimondo@unipv.it

**E-mail: limon@cartuja.us.es

***E-mail:m.lazar@tue.nl

****E-mail: eduardo@cartuja.us.es

The authors acknowledge the financial support of the $H Y C O N$ Network of Excellence, contract number FP6-IST-511368 and $M C Y T$-Spain (contracts DPI2007-66718-C04-01 and DPI2005-04568). 
a significant increase of the computational burden, an alternative approach consists in solving a min-max optimization problem. Specifically, in an open-loop formulation, the performance index is minimized with respect to the control sequence for the worst case, i.e. the disturbance sequence over the optimization horizon which maximizes the performance index. However, this solution is still unsatisfactory, since the minimization with respect to a single control profile does not solve the feasibility problem. This drawback can be avoided as in [4], where the MPC control law is applied to an already robustly stable system. Recently, a closed-loop formulation of the min-max controller has been proposed to reduce this conservativeness $[45,35,30]$. In this predictive control technique, a vector of feedback control policies is considered in the minimization of the cost in the worst disturbance case. This allows to take into account the reaction to the effect of the uncertainty in the predictions at expense of a practically untractable optimization problem.

In this context robust stability issues have been recently studied and some novel contributions on this topic have appeared in the literature $[45,15,19,29,16,8,32,22,23,25,31,27,20]$. In [34] two different cases are considered: when the uncertainty model does not depend on the state and when does depend on the state in such a way that the origin remains as equilibrium point. In order to analyze the stability properties of the uncertain system in the state dependent case, it is sufficient to use the notions of robust stability and stability margin [34]. On the contrary, a state independent disturbance asks for the use of Input-to-State Stability (ISS) and Input-to-State practical Stability (ISpS) concepts (see e.g. [48] and [18] for the continuous and the discrete time case respectively, and the further papers $[2,38,14,51])$ and sufficient stability conditions have been presented in [25, 23].

In order to apply the ISS property to MPC closed-loop systems (see e.g. [9, 23, 42, 31, 13, 25, 11, 21]), global results are in general not useful due to the presence of state and input constraints. On the other hand local results, see e.g. [18, 17], do not allow the analysis of the properties of the predictive control law in terms of its region of attraction. In [31] a regional version of the ISS has been used in the stability analysis of min-max MPC controllers.

\subsection{Purpose of this survey}

The objective of this paper is to summarize the results appearing in literature and to provide a general framework for synthesizing min-max MPC schemes with an a priori robust stability guarantee. To this aim, a general prediction model that covers a wide class of uncertainty modeling that includes bounded disturbances as well as state (and input) dependent disturbances (uncertainties) is introduced. This requires that the regional Input-to-State Stability (ISS) and practical ISS results are extended in order to cover both state dependent and state independent uncertainties. Moreover, Lyapunov-type sufficient conditions for the regional ISS and ISpS are presented for the considered class of systems; this constitutes the base of the stability analysis of the min-max MPC for the generalized prediction model.

Using this ISS tool, it is proven that if the terminal controller ensures ISS of the prediction system, w.r.t. the state independent part of the disturbance (i.e. the state dependent part of the disturbance remains in a certain stability margin) then the min-max MPC ensures that the closed loop system is ISpS maintaining the same stability margin. The practical nature of the stability is a consequence of the worst-case approach of the control action and causes the system to be ultimately bounded even if the real disturbances vanish.

In this survey paper, we concentrate on two different possible solutions to avoid this problem: the first one is based on an $\mathcal{H}_{\infty}$ like cost of the performance index (see e.g. [30, 29, 31, 16]), while the second one is based on a dual-mode strategy $[37,5,46,45,19,23]$. These solutions are extended to the generalized prediction model and it is shown that under fairly mild assumptions both controllers guarantee Input-to-State Stability.

Moreover, a nonlinear auxiliary control law, based on the one presented in [29], is proposed for the case of nonlinear systems affine in control (which are very usual). It is shown that a nonlinear auxiliary control law and the terminal penalty can be derived from the solution of the discrete-time $\mathcal{H}_{\infty}$ algebraic Riccati equation for the linearized system.

This survey paper is organized as follows: first, some notations used through the paper are presented and then the model of the plant and the control problem considered in this paper are stated. Section 4 is devoted 
to the regional version of ISS and ISpS in the case of the considered controlled systems. Based on this result, stability of the systems controlled by a min-max MPC is analyzed, considering different formulations: the standard, the $\mathcal{H}_{\infty}$ and the dual-mode approaches. In Section 6 , the proposed method for the calculation of a suitable auxiliary control law for a class of plants is presented. In the subsequent section, an example is given in order to compare the different strategies and, finally, some conclusions are derived. Some definitions and all the proofs of Theorems, Corollaries and Propositions are gathered in Appendix to improve readability.

\section{Notations and basic definitions}

Let $\mathcal{R}, \mathcal{R}_{\geq 0}, \mathcal{Z}$ and $\mathcal{Z}_{\geq 0}$ denote the real, the non-negative real, the integer and the non-negative integer numbers, respectively. Euclidean norm is denoted as $|\cdot|$. Given a signal $w$, the signal's sequence is denoted by $\mathbf{w} \triangleq\{w(0), w(1), \cdots\}$ where the cardinality of the sequence is inferred from the context. The set of sequences $w$, whose values belong to a compact set $W \subseteq \mathcal{R}^{m}$ is denoted by $\mathcal{M}_{W}$, while $W^{\text {sup }} \triangleq \sup _{w \in W}\{|w|\}$, $W^{\text {inf }} \triangleq \inf _{w \in W}\{|w|\}$. Moreover $\|w\| \triangleq \sup _{k \geq 0}\{|w(k)|\}$ and $\left\|w_{[\tau]}\right\| \triangleq \sup _{0 \leq k \leq \tau}\{|w(k)|\}$. The symbol id represents the identity function from $\mathcal{R}$ to $\mathcal{R}$, while $\gamma_{1} \circ \gamma_{2}$ is the composition of two functions $\gamma_{1}$ and $\gamma_{2}$ from $\mathcal{R}$ to $\mathcal{R}$. Given a set $A \subseteq \mathcal{R}^{n},|\zeta|_{\mathcal{A}} \triangleq \inf \{|\eta-\zeta|, \eta \in A\}$ is the point-to-set distance from $\zeta \in \mathcal{R}^{n}$ to $A$. The difference between two given sets $A \subseteq \mathcal{R}^{n}$ and $B \subseteq \mathcal{R}^{n}$ with $B \subseteq A$, is denoted by $A \backslash B \triangleq\{x: x \in A, x \notin B\}$. Given a closed set $A \subseteq \mathcal{R}^{n}, \partial A$ denotes the border of $A$. A function $\gamma: \mathcal{R}_{\geq 0} \rightarrow \mathcal{R}_{\geq 0}$ is of class $\mathcal{K}$ (or a "K $\mathcal{K}$-function") if it is continuous, positive definite and strictly increasing. A function $\gamma: \mathcal{R}_{\geq 0} \rightarrow \mathcal{R}_{\geq 0}$ is of class $\mathcal{K}_{\infty}$ if it is a $\mathcal{K}$-function and $\gamma(s) \rightarrow+\infty$ as $s \rightarrow+\infty$. A function $\beta: \mathcal{R}_{\geq_{0}} \times \mathcal{Z}_{\geq_{0}} \rightarrow \mathcal{R}_{\geq_{0}}$ is of class $\mathcal{K} \mathcal{L}$ if, for each fixed $t \geq 0, \beta(\cdot, t)$ is of class $\mathcal{K}$, for each fixed $s \geq 0, \beta(s, \cdot)$ is decreasing and $\beta(s, t) \rightarrow 0$ as $t \rightarrow \infty$.

\section{Problem statement}

In this paper it is assumed that the plant to be controlled is described by discrete-time nonlinear model:

$$
x(k+1)=f\left(x(k), u(k), d_{1}(k), d_{2}(k)\right), k \geq t, x(t)=\bar{x}
$$

where $x(k) \in \mathcal{R}^{n}$ is the system state, $u(k) \in \mathcal{R}^{m}$ is the current control vector and $d_{1}(k)$ and $d_{2}(k)$ are disturbances which model the uncertainties present in the model. This partition on the disturbance signals stems from its nature: $d_{1}(k) \in \mathcal{R}^{p}$ models a class of uncertainty which depends on the state and the control input while $d_{2}(k) \in \mathcal{R}^{q}$ models a class of uncertainty that does not depend neither on the state nor on the input signal.

Most of the models of nonlinear systems considers the uncertainty as bounded disturbances, that is, the only knowledge of the model mismatches is a bounded set where the error lies in. However, this representation may lead to conservative results when, as usually occurs, there exists a relationship between the model mismatch bounds and the state and input of the plant. In this case, this conservativeness would be reduced if this information were considered in the model of the plant by means of the proposed partition of the disturbance model.

In the following assumption, the considered structure of such models is formally presented.

\section{Assumption 1}

1. The uncertainty $d_{1}$ is such that

$$
d_{1}(k)=d_{1 \eta}(k) \eta(|(x(k), u(k))|)
$$

for all $k \geq 0$, where $\eta$ is a known $\mathcal{K}$-function and $d_{1 \eta} \in \mathcal{R}^{p}$ is modeled as confined in a compact set

$$
D_{1 \eta} \subset \mathcal{R}^{p}
$$

not necessarily including the origin with $D_{1 \eta}^{\text {inf }}$ and $D_{1 \eta}^{\text {sup }}$ known. 
2. The uncertainty $d_{2}$ is such that

$$
d_{2}(k) \in D_{2}
$$

for all $k \geq 0$, where $D_{2} \subset \mathcal{R}^{q}$ is a compact set containing the origin with $D_{2}^{\text {sup }}$ known.

3. The system has an equilibrium point at the origin, that is $f(0,0,0,0)=0$.

4. The control and state of the plant must fulfill the following constraints on the state and the input:

$$
\begin{aligned}
& u(k) \in U \\
& x(k) \in X
\end{aligned}
$$

where $X$ is a closed set and $U$ a compact set, both of them containing the origin.

5. The state of the plant $x(k)$ can be measured at each sample time.

The control objective consists in designing a control law $u=\kappa(x)$ such that it steers the system to (a neighborhood of) the origin fulfilling the constraints on the input and the state along the system evolution for any possible disturbance and yielding an optimal closed performance according to certain performance index.

This control problem is well studied in the literature and there exist a number of control techniques that could be used. However, among the existing solutions, one of the most successfully used control technique is the model predictive control in its min-max approach. This is due to its optimal formulation, its capability to ensure the robust constraint satisfaction and its stabilizing design [35].

A primary family of min-max MPC controllers determines the control law from the calculation of a sequence of control actions which ensures the constraint along the predicted trajectory of the plant for any possible uncertainty and minimizes the worst case performance index of the predicted evolution of the plant. These controllers are called open-loop min-max $M P C$ and it is well-known that this may lead to extremely conservative solutions. This is a consequence of the open loop nature of the predicted control actions.

If a control law is considered as decision variable in the optimization problem (instead of a control action), the solution results to be less conservative since the predicted controlled system reacts to the effect of the disturbance. The predictive controllers derived from this approach are called closed-loop min-max MPC controllers and can provide larger domain of attraction and a better closed-loop performance index. However, the optimization problem may be difficult (or even impossible) to be solved even for linear prediction models.

A practical solution, located between the open-loop and the closed-loop approach, is the so-called semifeedback formulation of the problem. In this case, control policies are considered as decision variables, but forcing a given structure of the control law. Thus, the decision variable of each control law is its set of defining parameters, yielding to an optimization problem similar to the open-loop case one.

In this paper, the considered control law is derived from a closed-loop min-max MPC formulation $\kappa(x)=$ $\kappa^{M P C}(x)$. Although, from a practical point of view, the control law is difficult to calculate, from a theoretical point of view makes sense since the closed-loop approach includes the open-loop and semi-feedback controllers, and these can be considered as particular cases. Thus, the stability results derived in the paper for closed-loop min-max MPC will be valid for the rest of formulations. It is worth remarking that this control law might be a discontinuous function of the state.

The resulting closed-loop system is given by

$$
x(k+1)=f\left(x(k), \kappa^{M P C}(x(k)), d_{1}(k), d_{2}(k)\right), k \geq t, x(t)=\bar{x}
$$

where the disturbance $d_{1}(k)$ is such that $d_{1}(k)=d_{1 \eta}(k) \eta\left(\left|\left(x(k), \kappa^{M P C}(x(k))\right)\right|\right)$ with $d_{1 \eta} \in D_{1 \eta}$, and the disturbance $d_{2}(k)$ is such that $d_{2}(k) \in D_{2}$. The control law should ensure that if the initial state is in a certain stability region, i.e. $\bar{x} \in X^{M P C}$, then the resulting evolution of the system fulfils the constraints, that is $x(k) \in X$ and $\kappa^{M P C}(x(k)) \in U$ for all $k \geq t$, for any possible evolution of the disturbance signals.

In the following section it is presented a suitable framework for the analysis of stability of such class of closed loop systems: the regional ISS. 


\section{Regional Input-to-state stability}

In this section the ISS framework for discrete-time autonomous nonlinear systems is presented and Lyapunov-like sufficient conditions are provided. This will be employed in this paper to study the behavior of perturbed nonlinear systems in closed-loop with min-max MPC controllers.

Consider a nonlinear discrete-time system described by

$$
x(k+1)=F\left(x(k), d_{1}(k), d_{2}(k)\right)
$$

where $x(k) \in \mathcal{R}^{n}$ is the state, $d_{1}(k) \in \mathcal{R}^{p}$ is the component of the uncertainty depending from the state and $d_{2}(k) \in \mathcal{R}^{q}$ is the other component of the uncertainty.

The transient of the system (7) with initial state $x(0)=\bar{x}$ and uncertainties $d_{1}$ and $d_{2}$ is denoted by $x\left(k, \bar{x}, \mathbf{d}_{\mathbf{1}}, \mathbf{d}_{\mathbf{2}}\right)$. Denote $\mathcal{M}_{D_{1}}$ the set of sequences $\mathbf{d}_{\mathbf{1}}$ such that $d_{1}(k) \in D_{1}(x(k)), \forall k \geq 0$.

Consider the following assumptions.

Assumption 2 The uncertainty $d_{1}$ is such that

$$
d_{1}(k) \in D_{1}(x(k)) \subset \mathcal{R}^{p}
$$

for all $k \geq 0$, where, for each $x, D_{1}(x)$ is closed and contains the origin. Moreover there exist a $\mathcal{K}$-function $\eta$ and a signal $d_{1 \eta} \in \mathcal{R}^{p}$, limited in a compact set $D_{1 \eta} \subset \mathcal{R}^{p}$ (not necessarily including the origin as an interior point) such that

$$
d_{1}(k)=d_{1 \eta}(k) \eta(|x(k)|)
$$

for all $k \geq 0$ and all $x \in \Xi$, where $\Xi \subseteq \mathcal{R}^{n}$ is a closed set containing the origin as an interior point.

The uncertainty $d_{2}$ is such that

$$
d_{2}(k) \in D_{2} \subset \mathcal{R}^{q}
$$

for all $k \geq 0$, where $D_{2}$ is a compact set containing the origin.

Assumption 3 The set $\mathcal{A} \subset \mathcal{R}^{n}$, containing the origin, is a zero-invariant set for the system (7), that is, a positively invariant set for the associated "undisturbed" system $x(k+1)=F(x(k), 0,0)$, that means

$$
F(x, 0,0) \in \mathcal{A}, \forall x \in \mathcal{A} .
$$

Consider the following definition.

Definition 1 (Robust positively invariant set) Suppose that Assumption 2 is satisfied. A set $\Xi \subseteq \mathcal{R}^{n}$ is a robust positively invariant set for system (7) if $F\left(x, d_{1}, d_{2}\right) \in \Xi$, for all $x \in \Xi$, all $d_{1} \in D_{1}(x)$ and all $d_{2} \in D_{2}$.

A regional version of ISpS $[48,18]$ is defined in the following.

Definition 2 (Regional ISpS in $\Xi$ ) Suppose that Assumption 2 is satisfied. Given a closed set $\Xi \subseteq \mathcal{R}^{n}$, including the origin as an interior point, system (7) with $\mathbf{d}_{\mathbf{1}} \in \mathcal{M}_{D_{1}}$ and $\mathbf{d}_{\mathbf{2}} \in \mathcal{M}_{D_{2}}$ is said to be ISpS (Inputto-State practical Stable) in $\Xi$ with respect to $d_{2}$ if $\Xi$ is robust positively invariant for (7) and if there exist a $\mathcal{K} \mathcal{L}$-function $\beta$, a $\mathcal{K}$-function $\gamma_{2}$ and a constant $c \geq 0$ such that

$$
\left|x\left(k, \bar{x}, \mathbf{d}_{\mathbf{1}}, \mathbf{d}_{\mathbf{2}}\right)\right| \leq \beta(|\bar{x}|, k)+\gamma_{2}\left(|| d_{2[k-1]}||\right)+c
$$

for all $\bar{x} \in \Xi$ and $k>0$. Whenever $c=0$ and (8) is satisfied, the system (7) is said to be ISS (Input-to-State Stable) in $\Xi$ with respect to $d_{2}$. 
In many applications it is of interest to study the stability with respect to an invariant set $\mathcal{A}$, where $\mathcal{A}$ in general does not consist of a single point. The "set" version of the ISS property (also known as "compactISS") was originally proposed in [49]. Suppose that Assumption 3 is satisfied. A regional version of ISS with respect to $\mathcal{A}[47,10]$ is given in Appendix. In Proposition VI.3 of [48] and in Definition 2.4 of [50], it is shown, for the continuous-time case, that concept of ISpS is equivalent to concept of ISS with respect to $\mathcal{A}$. In this case, the constant $c$ of equation (8) is $c \triangleq \mathcal{A}^{\text {sup }}$. The same relation holds for discrete-time case.

The concepts of LpS, UpAG in $\Xi$, LS and UAG in $\Xi$ with respect to $\mathcal{A}$ are given in Appendix. As for the ISpS property, the concepts of LpS and UpAG are equivalent to the concepts of LS and UAG with respect to $\mathcal{A}$. On the base of the results obtained in $[50,48,10]$, it is possible to give the following equivalence result.

Theorem 1 Suppose that Assumption 3 is satisfied. Consider system (7). The following properties are equivalent:

a) $\operatorname{ISpS}$ in $\Xi$;

b) ISS in $\Xi$ with respect to $\mathcal{A}$;

c) UpAG in $\Xi$ and $L p S$;

d) $U A G$ in $\Xi$ with respect to $\mathcal{A}$ and $L S$ with respect to $\mathcal{A}$.

Remark 1 The assumption that $\Xi$ is robust positively invariant could render the definitions of ISpS and UpAG in $\Xi$ trivials. In fact ISS in $\Xi$ with respect to $\Xi$ and ISpS in $\Xi$ with $\mathcal{A} \equiv \Xi$ are always satisfied. However, if in the ISpS property, $c$ is shown to be smaller than $\Xi^{\text {sup }}$, and in the ISS the set $\mathcal{A}$ is shown to be smaller than $\Xi$, then the ISpS in $\Xi$ (or the ISS in $\Xi$ with respect to $\mathcal{A}$ ) give more information than the solely robust positively invariance of $\Xi$.

Regional ISpS will be now associated to the existence of a suitable Lyapunov function (in general, a priori non-smooth) with respect to $d_{2}$. A sufficient condition, that extends the ISS results of [31], using the results of [25], is introduced. In order to clarify the relation between the sets introduced in the following definition see Figure 1.

Definition 3 (ISpS-Lyapunov function in $\Xi$ ) Under Assumption 2, a function $V: \mathcal{R}^{n} \rightarrow \mathcal{R}_{\geq} 0$ is called an ISpS-Lyapunov function in $\Xi$ for system (7) with respect to $d_{2}$, if:

1) $\Xi$ is a closed robust positively invariant set including the origin as an interior point

2) there exist a compact set $\Omega \subseteq \Xi$ (including the origin as an interior point), a pair of suitable $\mathcal{K}_{\infty^{-}}$ functions $\alpha_{1}, \alpha_{2}$ and a constant $c_{1} \geq 0$ such that:

$$
\begin{gathered}
V(x) \geq \alpha_{1}(|x|), \forall x \in \Xi \\
V(x) \leq \alpha_{2}(|x|)+c_{1}, \forall x \in \Omega
\end{gathered}
$$

3) there exists a suitable $\mathcal{K}_{\infty}$-function $\alpha_{3}$, a $\mathcal{K}$-function $\lambda_{2}$ and a constant $c_{2} \geq 0$ such that:

$$
\begin{aligned}
\Delta V(x) \triangleq & V\left(F\left(x, d_{1}, d_{2}\right)\right)-V(x) \\
\leq & -\alpha_{3}(|x|)+\lambda_{2}\left(\left|d_{2}\right|\right)+c_{2}, \\
& \forall x \in \Xi, \forall d_{1} \in D_{1}(x), \forall d_{2} \in D_{2}
\end{aligned}
$$


4) there exist suitable $\mathcal{K}_{\infty}$-functions $\zeta$ and $\rho$ (with $\rho$ such that $(i d-\rho)$ is a $\mathcal{K}_{\infty}$-function) and a suitable constant $c_{\theta}>0$, such that there exists a nonempty compact set $\Theta \subset\left\{x: x \in \Omega, d(x, \delta \Omega)>c_{\theta}\right\}$ (including the origin as an interior point) defined as follows:

$$
\Theta \triangleq\left\{x: V(x) \leq b\left(\lambda_{2}\left(D_{2}^{\text {sup }}\right)+c_{3}\right)\right\}
$$

where $b \triangleq \alpha_{4}^{-1} \circ \rho^{-1}$, with $\alpha_{4} \triangleq \underline{\alpha}_{3} \circ \bar{\alpha}_{2}^{-1}, \underline{\alpha}_{3}(s) \triangleq \min \left(\alpha_{3}(s / 2), \zeta(s / 2)\right), \bar{\alpha}_{2} \triangleq \alpha_{2}(s)+s, c_{3} \triangleq c_{2}+\zeta\left(c_{1}\right)$.

A function $V: \mathcal{R}^{n} \rightarrow \mathcal{R}_{\geq} 0$ is called an ISS-Lyapunov function in $\Xi$ if it is an ISpS-Lyapunov function in $\Xi$ with $c_{1}=c_{2}=0$ (see [31]).

Figure 1: Example of sets satisfying Definition 3.

A sufficient condition for regional ISpS of system (7), that extends the ISS results of [31] using the results of [25], can now be stated.

Theorem 2 If system (7) admits an ISpS-Lyapunov function in $\Xi$ with respect to $d_{2}$, then it is ISpS in $\Xi$ with respect to $d_{2}$ and $\lim _{k \rightarrow \infty}\left|x\left(k, \bar{x}, \mathbf{d}_{\mathbf{1}}, \mathbf{d}_{\mathbf{2}}\right)\right|_{\Theta}=0$.

Remark 2 In the proof of Theorem 2, it is shown that, in order to prove the ISpS, the upper bound (10) in a local region is sufficient. However, this could be a limitation due to (12). In fact the uncertainty should be such that $\Theta \subseteq \Omega$. In order to enlarge the set of admissible uncertainty it could be useful to find an upper bound in a region $\Omega_{1} \supseteq \Omega$ as suggested in [25, 23] for the MPC case. However this idea can either enlarge or restrict the set of admissible uncertainty since $\Omega_{1} \supseteq \Omega$ but the upper bound could be more conservative.

Remark 3 Note that for a generic disturbance $d_{1}$, condition 3) of Definition 3 should be:

there exists a suitable $\mathcal{K}_{\infty}$-function $\alpha_{3}$, a pair of $\mathcal{K}$-functions $\lambda_{1}$ and $\lambda_{2}$ and a constant $c_{2} \geq 0$ such that:

$$
\begin{aligned}
\Delta V(x) \triangleq & V\left(F\left(x, d_{1}, d_{2}\right)\right)-V(x) \leq-\alpha(|x|)+\lambda_{1}\left(\left|d_{1}\right|\right)+\lambda_{2}\left(\left|d_{2}\right|\right)+c_{2}, \\
& \forall x \in \Xi, \forall d_{1} \in D_{1}(x), \forall d_{2} \in D_{2} .
\end{aligned}
$$

However, in view of Assumption 2, since $d_{1}$ is a function of $x$, the term $\lambda_{1}\left(d_{1}(x)\right)$ is incorporated in $-\alpha_{3}(|x|)$. In order to satisfy the condition that $\alpha_{3}$ is a $\mathcal{K}_{\infty}$-function, it is necessary that the $\mathcal{K}_{\infty}$-function $\alpha$ in (13) compensates the effect of the disturbance $d_{1}$. This means that system (7) must have a stability margin: under Assumption 2, it is required that

$$
-\alpha(|x|)+\lambda_{1}\left(\left|d_{1}\right|\right)=-\alpha(|x|)+\lambda_{1}\left(\left|d_{1 \eta}\right| \eta(|x|)\right) \leq-\alpha(|x|)+\lambda_{1}\left(D_{1 \eta}^{\text {sup }} \eta(x)\right) \triangleq-\alpha_{3}(|x|)
$$

with $\alpha_{3} \in \mathcal{K}_{\infty}$-function.

\section{Min-max model predictive control}

This section presents new results that allows the presentation of previous results in a unified framework. Firstly, the formulation of the closed-loop min-max control law is presented. Then, the stability of different approaches of this control technique is studied, deriving sufficient conditions and generalizing existing results.

As it was claimed in Section 3, the control law derived by means of a closed-loop min-max MPC considers a vector of feedback control policies $\kappa_{t, t+N-1}=\left[\kappa_{0}(x(t)), \kappa_{1}(x(t+1)), \cdots, \kappa_{N-1}(x(t+N-1))\right]$ in the minimization of the cost in the worst disturbance case. This optimization problem can be posed as the following Finite Horizon Closed-Loop Game ( $F H C L G)$. 
Definition 4 (FHCLG) Suppose that Assumption 1 is satisfied. Given the positive integer $N$, the stage cost $l$, the terminal penalty $V_{f}$ and the terminal set $X_{f}$, the $F H C L G$ problem consists in minimizing, with respect to $\kappa_{t, t+N-1}$ and maximizing with respect to $d_{1[t, t+N-1]}$ and $d_{2[t, t+N-1]}$ the cost function

$$
J\left(\bar{x}, \kappa_{t, t+N-1}, d_{1[t, t+N-1]}, d_{2[t, t+N-1]}, N\right) \triangleq \sum_{k=t}^{t+N-1} l\left(x(k), u(k), d_{1}(k), d_{2}(k)\right)+V_{f}(x(t+N))
$$

subject to

i) the state dynamics (1), with $x(t)=\bar{x}$;

ii) the constraints (3)-(6), $k \in[t, t+N-1]$;

iii) the terminal constraint $x(t+N) \in X_{f}$.

Letting $\kappa_{t, t+N-1}^{o}, d_{1[t, t+N-1]}^{o}, d_{2[t, t+N-1]}^{o}$ be the solution of the $F H C L G$, according to the Receding Horizon $(R H)$ paradigm, the feedback control law $u=\kappa^{M P C}(x)$ is obtained by setting

$$
\kappa^{M P C}(x)=\kappa_{0}^{o}(x)
$$

where $\kappa_{0}^{o}(x)$ is the first element of $\kappa_{t, t+N-1}^{o}$.

The parameters of the controller are the prediction horizon $N$, the stage cost function, the terminal cost function and the terminal region. The stage cost defines the performance index to optimize and must satisfy following assumption.

Assumption 4 The stage cost $l(\cdot)$ is such that $l(0,0,0,0)=0$ and $l\left(x, u, d_{1}, d_{2}\right) \geq \alpha_{l}(|x|)-\alpha_{d}\left(\left|d_{2}\right|\right)$ where $\alpha_{l}$ and $\alpha_{d}$ are $\mathcal{K}_{\infty}$-functions.

As it is standard in MPC [35], the terminal ingredients are added to provide closed-loop stability as it can be seen in the following section.

\subsection{Stability}

In this section we provide tools for analyzing stability of closed-loop min-max MPC systems. Firstly, it will be shown that, when persistent disturbances are present, the standard min-max approach can only guarantee ISpS. Secondly, two different solutions for overcoming this problem and guaranteing ISS of the min-max MPC closed-loop system are given: the first one is derived using a particular design of the stage cost of the performance index, while the second one is based on a dual-mode strategy.

The presence of constraints in the system may limit the domain of attraction of the controlled system to a certain region. This region is introduced in the following definition.

Definition 5 (Robust invariant region) Suppose that Assumption 1 is satisfied. Given a control law $u=\kappa(x), \bar{X} \subseteq X$ is a robust invariant region for the closed-loop system (1) with $u(k)=\kappa(x(k))$, if $\bar{x} \in \bar{X}$ implies $x(k) \in \bar{X}$ and $\kappa(x(k)) \in U, \forall k \geq t$.

In order to derive the main stability properties, the optimal value of the performance index, i.e.

$$
V(x, N) \triangleq J\left(\bar{x}, \kappa_{t, t+N-1}^{o}, d_{1[t, t+N-1]}^{o}, d_{2[t, t+N-1]}^{o}, N\right),
$$

is employed as an ISpS-Lyapunov function. Furthermore, the following assumptions are introduced.

Assumption 5 The design parameters $V_{f}, X_{f}$ are such that, given an auxiliary control law $\kappa_{f}$ 
1) $X_{f} \subseteq X, X_{f}$ closed, $0 \in X_{f}$;

2) $\kappa_{f}(x) \in U,\left|\kappa_{f}(x)\right| \leq L_{f}|x|, \forall x \in X_{f}$, where $L_{f}>0$;

3) $f\left(x, \kappa_{f}(x), d_{1}, d_{2}\right) \in X_{f}, \forall x \in X_{f}, \forall d_{1 \eta} \in D_{1 \eta}, \forall d_{2} \in D_{2}$;

4) $\alpha_{V_{f}}(|x|) \leq V_{f}(x) \leq \beta_{V_{f}}(|x|), \forall x \in X_{f}$, where $\alpha_{V_{f}}$ and $\beta_{V_{f}}$ are $\mathcal{K}_{\infty}$-functions;

5) $V_{f}\left(f\left(x, \kappa_{f}(x), d_{1}, d_{2}\right)-V_{f}(x) \leq-l\left(x, \kappa_{f}(x), d_{1}, d_{2}\right)+\varrho\left(\left|d_{2}\right|\right)\right.$, $\forall x \in X_{f}, \forall d_{1 \eta} \in D_{1 \eta}, \forall d_{2} \in D_{2}$, where $\varrho$ is a $\mathcal{K}_{\infty}$-function.

Assumption 5 implies that the closed-loop system formed by (1) and $u(k)=\kappa_{f}(x)$, is ISS in $X_{f}\left(V_{f}\right.$ is an ISS-Lyapunov function in $X_{f}$ ).

Remark 4 If the feedback policies $\kappa_{i}(x), i=0, \ldots, N-1$ are restricted to belong to a particular class of functions then also $\kappa_{f}$ must belong to this class. This motivates the difficulty to guarantee closed-loop stability if optimization is performed with respect to open-loop strategies [4]. In fact, Assumption 5 should be verified with $\kappa_{f}(x)=0$. On the contrary, a natural choice, when semi-feedback controllers are used, is to include the auxiliary control law among the regressors (see the example in Section 7).

Assumption 6 Let $X^{M P C}(N)$ denote the set of states for which a solution of the FHCLG problem exists. Let $\Omega=X_{f}, \alpha_{1}=\alpha_{l}, \alpha_{2}=\beta_{V_{f}}, c_{1}=N \varrho\left(D_{2}^{\text {sup }}\right), \alpha_{3}=\alpha_{l}, \lambda=\alpha_{d}, c_{2}=\varrho\left(D_{2}^{\text {sup }}\right)$. The set $D_{2}$ is such that the set $\Theta$ (depending from $D_{2}^{\text {sup }}$ ), defined in (12), is strictly contained in $\Omega$.

Consider now the following stability result for the classic min-max MPC problem.

Theorem 3 Under Assumptions 1, 4-6, the closed-loop system formed by (1) and (15) is ISpS with respect to $d_{2}$ with robust invariant region $X^{M P C}(N)$.

Remark 5 Following the result in [46] for standard NMPC, assuming that an initial feasible solution of the FHCLG is available, it is possible to show that it is not necessary to obtain the global optimum solution of the FHCLG in order to guarantee the ISpS (or the ISS) of the closed-loop system. In fact the vector of feedback control policies $\tilde{\kappa}_{1, N} \triangleq\left[\tilde{\kappa}_{0, N-1} \kappa_{f}\right]$, where $\tilde{\kappa}_{0, N-1}$ is the possible sub-optimal solution obtained at the previous step, is an available feasible solution that guarantees ISpS or ISS. Indeed this sequence is such that the value function satisfies (11). The only requirement on the possible sub-optimal solution is to be not worst than $\tilde{\kappa}_{1, N}$. On the contrary, the applicability of a sub-optimal solution of the maximization of the FHCLG is still an open issue [1, 40].

The previous theorem formulated for the general case of standard min-max MPC states that only ISpS is guaranteed for the resulting closed-loop system, irrespective of the fact that the disturbances may vanish in reality. However, when this is the case, it would be preferable that the closed-loop system is ISS, so that nominal asymptotic stability is recovered when disturbances are no longer active.

In the following subsections we present some ingredients, in the form of assumptions on the type of disturbances or the min-max MPC cost function, that make it possible to establish ISS, instead of ISpS, of closed-loop min-max MPC systems. 


\subsubsection{Standard min-max with only state dependent uncertainty}

Consider the case when system (1) is affected only by the uncertainty $d_{1}$ satisfying (2) (it is known that $\left.d_{2}(k)=0, \forall k \geq 0\right)$. This assumption led to the result published in [34], which is stated in the following theorem.

Theorem 4 [34] Consider that $d_{2}=0$. Under Assumptions 1, 4-6, the origin of the closed-loop system formed by (1) and (15) is robustly asymptotically stable with domain of attraction $X^{M P C}(N)$.

Remark 6 Note that Assumption 5 states that control law $u=\kappa_{f}(x)$ is designed in such a way that the closed-loop system has a stability margin in $X_{f}$. Moreover, note that the robustness of the auxiliary control law is translated to the $M P C$, that is, the min-max $M P C$ controller extends to $X^{M P C}(N)$ the stability margin provided by the auxiliary control law.

Next, we will consider the more challenging case when both state dependent and state independent uncertainties are present.

\subsubsection{Standard min-max with state independent uncertainty}

Consider the case system (1) is affected by both uncertainties of the type $d_{1}(k)$ and of the type $d_{2}(k)$. A new condition on the stage cost (standard min-max stage cost) is introduced.

Assumption 7 The stage cost $l(x, u)$ is disturbance independent and such that $l(0,0)=0$ and $l(x, u) \geq$ $\alpha_{l}(|x|)$, where $\alpha_{l}$ is a $\mathcal{K}_{\infty}$-function.

Under the above assumption, the following ISpS result (which can be recovered also from Theorem 3) for min-max MPC was obtained in [25].

Corollary 1 [25] Under Assumptions 1, 5-7, the closed-loop system formed by (1) and (15) is ISpS with respect to $d_{2}$ with robust invariant region $X^{M P C}(N)$.

Note that, even if the auxiliary control strategy guarantees ISS, only ISpS can be established for the minmax MPC closed-loop system. As already mentioned, this is not a desirable property, as the employed control design method prevents that closed-loop asymptotic stability is attained when there are no disturbances active.

In the followings we will discuss two solutions for solving this problem of standard min-max MPC. The first solution employs a particular design of the stage cost such that Assumption 5 can be satisfied also with $\varrho \equiv 0$, leading, for example, to the well-known $\mathcal{H}_{\infty}$ strategy. The second solution exploits the ISS property of the auxiliary control law via a dual-mode strategy to establish ISS of the dual-mode min-max MPC closed-loop system.

\subsection{3 $\mathcal{H}_{\infty}$ strategy}

The proof of Corollary 1 clearly illustrates that the difficulty of proving ISS of min-max MPC, which is attempted in Theorem 3, is related to the terms $c_{1}$ and $c_{2}$ that are depending on the $\mathcal{K}_{\infty}$-function $\varrho$ defined in Assumption 5 and whose necessity is related to the stage cost considered in the standard min-max MPC optimization problem.

This observation leads to the following new condition on the stage cost, which will turn out to be sufficient for proving ISS of the corresponding min-max MPC closed-loop system. 
Assumption 8 The stage cost is composed by the functions $l_{x}: \mathcal{R}^{n} \times \mathcal{R}^{m} \times \mathcal{R}^{p} \rightarrow \mathcal{R}$ and $l_{d}: \mathcal{R}^{q} \times \mathcal{R}^{m} \rightarrow \mathcal{R}$ as follows

$$
l\left(x, u, d_{1}, d_{2}\right)=l_{x}\left(x, u, d_{1}\right)-l_{d}\left(d_{2}\right)
$$

and satisfies Assumption 4.

If Assumption 8 is satisfied, as it will be shown in Section 6, then Assumption 5 can be satisfied also with $\varrho \equiv 0$. Then, using the proof of Theorem 3, ISS of the closed-loop system can be guaranteed, as established by the following result.

Corollary 2 [31] Under Assumptions 1, 5, 6, 8 with $\varrho \equiv 0$, the closed-loop system formed by (1) and (15) is ISS with respect to $d_{2}$ with robust invariant region $X^{M P C}(N)$.

The above result shows that by adding a new term to the stage cost, which depends solely on the disturbance signal, ISS of the resulting min-max MPC closed-loop system can be attained. It remains to be explored how the modified cost function affects the solvability of the min-max optimization problem and whether standard min-max MPC solvers can still be employed.

Next, we present a dual-mode strategy for guaranteeing ISS of min-max MPC, which relies on the same cost function as the one used in standard min-max MPC.

\subsubsection{Dual-mode strategy}

Sufficient conditions for input-to-state stability of nonlinear discrete-time systems in closed-loop with dual-mode min-max MPC controllers were recently developed in [23]. Therein, only state independent uncertainties were considered. In this section we exploit the results presented in [23] and we use Theorem 2 to apply the dual-mode approach to the more general class of uncertainties considered in this paper.

First, let us recall the classical dual-mode strategy. In dual-mode MPC, the receding horizon controller (15) is employed outside $X_{f}$ and the auxiliary control law $\kappa_{f}$ is used inside $X_{f}$, i.e.

$$
u^{D M} \triangleq \begin{cases}\kappa^{M P C}(x) & \text { if } x \in X^{M P C}(N) \backslash X_{f} \\ \kappa_{f}(x) & \text { if } x \in X_{f}\end{cases}
$$

Next, we present the ISS result for dual-mode min-max MPC with standard cost function.

Theorem 5 Under Assumptions 1, 4-6, the closed-loop system formed by (1) and (16) is ISS with respect to $d_{2}$ with robust invariant region $X^{M P C}(N)$.

So far, we have presented two methods for establishing ISS of min-max MPC. However, both these methods rely on a specific cost function that must satisfy certain assumptions. Therefore, the computation of suitable cost functions is equally important for synthesizing min-max MPC schemes with an a priori ISS guarantee. A solution that applies to nonlinear systems affine in control is presented in the next section.

\section{The auxiliary control law}

In this section it is shown that, if nonlinear input affine systems are considered, a nonlinear control law $u=\hat{\kappa}(x)$ satisfying Assumption 5 can be derived by the solution of the $\mathcal{H}_{\infty}$ control problem for the linearized system. In this respect, consider the system

$$
\begin{aligned}
& x(k+1)=f_{1}(x(k))+f_{2}(x(k)) u(k)+f_{3}(x(k)) w(k) \\
& z(k)=\left[\begin{array}{l}
h_{1}(x(k)) \\
u
\end{array}\right]
\end{aligned}
$$


where $w=\left[\begin{array}{ll}d_{1} & d_{2}\end{array}\right]^{\prime}, f_{1}, f_{2}, f_{3}$ and $h_{1}$ are $C^{2}$ functions with $f_{1}(0)=0$ and $h_{1}(0)=0$. For convenience, we represent the corresponding discrete-time linearized system as

$$
\begin{aligned}
& x(k+1)=F_{1} x(k)+F_{2} u(k)+F_{3} w(k) \\
& z(k)=\left[\begin{array}{l}
H_{1} x(k) \\
u
\end{array}\right]
\end{aligned}
$$

where $F_{1}=\left.\frac{\partial f_{1}}{\partial x}\right|_{x=0}, F_{2}=f_{2}(0), F_{3}=f_{3}(0), H_{1}=\left.\frac{\partial h_{1}}{\partial x}\right|_{x=0}$. Given a square $n \times n$ matrix $P$, define also the symmetric matrix

$$
R=R(P)=\left[\begin{array}{ll}
R_{11} & R_{12} \\
R_{21} & R_{22}
\end{array}\right]
$$

where

$$
\begin{aligned}
& R_{11}=F_{2}^{\prime} P F_{2}+I \\
& R_{12}=R_{21}^{\prime}=F_{2}^{\prime} P F_{3} \\
& R_{22}=F_{3}^{\prime} P F_{3}-\gamma^{2} I
\end{aligned}
$$

and the quadratic function

$$
V_{f}(x)=x^{\prime} P x .
$$

Proposition 1 Suppose that Assumptions 1 is satisfied. Suppose there exists a positive definite matrix $P$ such that

(i) $R_{22}<0$

(ii) $-P+F_{1}^{\prime} P F_{1}+H_{1}^{\prime} H_{1}-F_{1}^{\prime} P\left[\begin{array}{ll}F_{2} & F_{3}\end{array}\right] R^{-1}\left[\begin{array}{ll}F_{2} & F_{3}\end{array}\right]^{\prime} P F_{1}<0$

and that

(iii) $\left|d_{1}\right|^{2}=\left|d_{1 \eta} \eta(|(x, u)|)\right|^{2} \leq K_{d x}|x|^{2}+K_{d u}|u|^{2}$, with $K_{d x} \geq 0$ and $K_{d u} \geq 0$.

Then, there exist sets $D_{1}(x)$ and $D_{2}$ such that $\forall w \in \bar{W}_{n e}=D_{1}(x) \times D_{2}$ the control law $u=\kappa^{*}(x)$ where

$$
\left[\begin{array}{l}
\kappa^{*}(x) \\
\xi^{*}(x)
\end{array}\right]=-R(x)^{-1}\left[\begin{array}{ll}
f_{2}(x) & f_{3}(x)
\end{array}\right]^{\prime} P f_{1}(x)
$$

with

$$
R(x)=\left[\begin{array}{ll}
f_{2}(x)^{\prime} P f_{2}(x)+I & f_{2}(x)^{\prime} P f_{3}(x) \\
f_{3}(x)^{\prime} P f_{2}(x) & f_{3}(x)^{\prime} P f_{3}(x)-\gamma^{2} I
\end{array}\right]=\left[\begin{array}{ll}
r_{11}(x) & r_{12}(x) \\
r_{21}(x) & r_{22}(x)
\end{array}\right]
$$

satisfies Assumption 5 with stage cost

(a) $l\left(x, u, d_{1}, d_{2}\right)=|z|^{2}-\gamma^{2}|w|^{2}$ and $\varrho \equiv 0$

or

(b) $l\left(x, u, d_{1}, d_{2}\right)=\left|z_{l}\right|^{2}$ with $z_{l}=\left[\begin{array}{l}h_{l}(x) \\ u\end{array}\right]$, where $h_{l}$ is such that $h_{1}(x)^{\prime} h_{1}(x) \geq h_{l}(x)^{\prime} h_{l}(x)+a|x|^{2}+c|x|^{2}$, $a \triangleq \gamma^{2} K_{d x}, b \triangleq \gamma^{2} K_{d u}, c \triangleq b L_{f}^{2}$ and $\varrho(s) \triangleq \gamma^{2}|s|^{2}$

and

$$
X_{f} \triangleq\left\{x: x^{\prime} P x \leq \alpha\right\} \subseteq X,
$$

where $\alpha$ is a finite positive constant.

Remark $7 P$ can be computed by solving a discrete-time $\mathcal{H}_{\infty}$ algebraic Riccati equation. 


\section{Example}

In this section, the MPC law introduced in the paper is applied to a cart with mass $M$ moving on a plane (the model is the same of the paper [29]). This carriage (see Figure 2) is attached to the wall via a spring with elastic constant $k$ given by $k=k_{0} e^{-x_{1}}$, where $x_{1}$ is the displacement of the carriage from the equilibrium position associated with the external force $u=0$ and the external disturbance force (wind force) $d_{2}=0$. Finally a damper with damping factor $h_{d}$ affects the system in a resistive way. The model of the

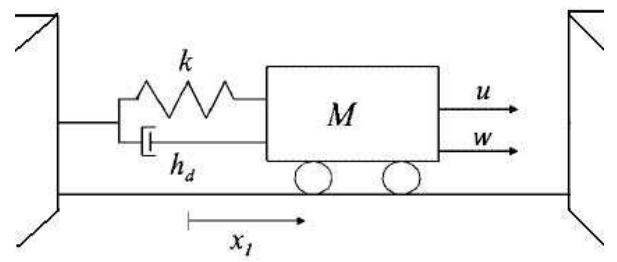

Figure 2: Cart and spring-damper example.

system is given by the following continuous-time state space nonlinear model

$$
\left\{\begin{array}{l}
\dot{x}_{1}(t)=x_{2}(t) \\
\dot{x}_{2}(t)=-\frac{k_{0}}{M} e^{-x_{1}(t)} x_{1}(t)-\frac{h_{d}}{M} x_{2}(t)+\frac{u(t)}{M}+\frac{d_{2}(t)}{M}
\end{array}\right.
$$

where $x_{2}$ is the carriage velocity. The parameters of the system are $M=1 \mathrm{~kg}, k_{0}=0.33 \frac{\mathrm{N}}{\mathrm{m}}$, while the damping factor in not well known and is given by $h_{d}=\bar{h}_{d}+d_{1 \eta}(t)$, where $\bar{h}_{d}=1.1 \frac{\mathrm{Ns}}{\mathrm{m}}$ and $\left|d_{1 \eta}(t)\right| \leq 0.1$. Wind force is limited: $-0.2 \leq d_{2} \leq 0.4$. The system can be rewritten as

$$
\left\{\begin{array}{l}
\dot{x}_{1}(t)=x_{2}(t) \\
\dot{x}_{2}(t)=-\frac{k_{0}}{M} e^{-x_{1}(t)} x_{1}(t)-\frac{\bar{h}_{d}}{M} x_{2}(t)+\frac{u(t)}{M}-\frac{d_{1 \eta}(t)}{M} x_{2}(t)+\frac{d_{2}(t)}{M}
\end{array}\right.
$$

The state and control variables have to satisfy the following constraints $|u| \leq 4.5 N,\left|x_{1}\right| \leq 2.65 \mathrm{~m}$. An Euler approximation of the system with sampling time $T_{c}=0.4 s$ is given by

$$
\left\{\begin{aligned}
x_{1}(k+1)= & x_{1}(k)+T_{c} x_{2}(k) \\
x_{2}(k+1)= & -T_{c} \frac{k_{0}}{M} e^{-x_{1}(k)} x_{1}(k)+x_{2}(k)-T_{c} \frac{\bar{h}_{d}}{M} x_{2}(k) \\
& +T_{c} \frac{u(k)}{M}-T_{c} \frac{d_{1 \eta}(k)}{M} x_{2}(k)+T_{c} \frac{d_{2}(k)}{M}
\end{aligned}\right.
$$

which is a discrete-time nonlinear system. The system can be rewritten as

$$
\left[\begin{array}{l}
x_{1}(k+1) \\
x_{2}(k+1)
\end{array}\right]=\underbrace{\left[\begin{array}{ll}
1 & T_{c} \\
-T_{c} \frac{k_{0}}{M} e^{-x_{1}(k)} & 1-T_{c} \frac{\bar{h}_{d}}{M}
\end{array}\right]\left[\begin{array}{l}
x_{1}(k) \\
x_{2}(k)
\end{array}\right]}_{f_{1}(x(k))}+\underbrace{\left[\begin{array}{c}
0 \\
\frac{T_{c}}{M}
\end{array}\right]}_{F_{2}} u(k)+\underbrace{\left[\begin{array}{cc}
0 & 0 \\
-\frac{T_{c}}{M} & \frac{T_{c}}{M}
\end{array}\right]}_{F_{3}} \underbrace{\left[\begin{array}{c}
d_{1}(k) \\
d_{2}(k)
\end{array}\right]}_{w(k)}
$$

where $d_{1}(k)=d_{1 \eta}(k) x_{2}(k)$. Disturbance $d_{1}(k)$ satisfies point $($ iii $)$ of Proposition 1 with $K_{d x}=0.01$ and $K_{d u}=0$. Let choose $l\left(x, u, d_{1}, d_{2}\right)=\left|z_{l}\right|^{2}-\gamma^{2}|w|^{2}$ and $l(x, u)=\left|z_{l}\right|^{2}$ where

$$
z_{l}=\left[\begin{array}{l}
h_{l}(x) \\
u
\end{array}\right]=[\overbrace{\left[\begin{array}{cc}
q_{1 l} & 0 \\
0 & q_{2 l}
\end{array}\right]}^{H_{L}}\left[\begin{array}{l}
x_{1} \\
x_{2}
\end{array}\right]]
$$

with $q_{1 l}=1$ and $q_{2 l}=1$ and $\gamma=3$. The auxiliary control law is obtained as described in Section 6 with

$$
z=\left[\begin{array}{l}
h_{1}(x) \\
u
\end{array}\right]=[\overbrace{\left[\begin{array}{ll}
q_{1} & 0 \\
0 & q_{2}
\end{array}\right]}^{H_{1}}\left[\begin{array}{l}
x_{1} \\
x_{2}
\end{array}\right]]
$$


with $q_{1}=1.1$ and $q_{2}=1.1$. Note that, as required at point $\left.b\right)$ of Proposition $1, h_{1}(x)^{\prime} h_{1}(x) \geq h_{l}(x)^{\prime} h_{l}(x)+$ $a|x|^{2}+c|x|^{2}$ with $a=\gamma^{2} K_{d x}=0.09, b=\gamma^{2} K_{d u}=0, c=b L_{f}^{2}=0$, where $L_{f}=2$. In fact

$$
\begin{aligned}
q_{1}^{2} x_{1}^{2}+q_{2}^{2} x_{2}^{2} & \geq q_{1 l}^{2} x_{1}^{2}+q_{2 l}^{2} x_{2}^{2}+a x_{1}^{2}+a x_{2}^{2} \\
1.21 x_{1}^{2}+1.21 x_{2}^{2} & \geq x_{1}^{2}+x_{2}^{2}+0.09 x_{1}^{2}+0.09 x_{2}^{2} .
\end{aligned}
$$

The auxiliary control law is given by

$$
\kappa_{f}(x)=-\left[\begin{array}{lll}
1 & 0 & 0
\end{array}\right] R^{-1}\left[\begin{array}{c}
F_{2} \\
F_{3}
\end{array}\right] P f_{1}(x)=-[0.87831 .1204] f_{1}(x)
$$

where

$$
P=\left[\begin{array}{ll}
7.0814 & 3.3708 \\
3.3708 & 4.2998
\end{array}\right]
$$

is computed solving a discrete time $\mathcal{H}_{\infty}$ algebraic Riccati equation

$$
P=F_{1}^{\prime} P F_{1}+H_{2}^{\prime} H_{2}-F_{1}^{\prime} P\left[\begin{array}{ll}
F_{2} & F_{3}
\end{array}\right] R^{-1}\left[\begin{array}{ll}
F_{2} & F_{3}
\end{array}\right]^{\prime} P F_{1}
$$

with

$$
H_{2}^{\prime} H_{2}=1.2 H_{1}^{\prime} H_{1}
$$

in order to satisfy inequality (ii) of Proposition 1. Matrix $P$ satisfies inequality $(i)$ of Proposition 1 . The terminal penalty is given by $V_{f}=x^{\prime} P x$. The auxiliary control law satisfies Assumption 5 , for the stages cost chosen, in the region $X_{f} \triangleq\left\{x: x^{\prime} P x \leq 4.7\right\}$. Region $X_{f}$ has been obtained numerically. The length of horizon is $N=4$. The policies $\kappa_{i}(x)$ are functions of the form $\kappa_{i}(x)=\alpha_{i} \kappa_{f}(x)+\beta_{i}\left(x_{1}^{2}+x_{2}^{2}\right)+\gamma_{i}$. Figure $3(\mathrm{a})$ and $3(\mathrm{~b})$ show the time evolution of position and velocity of the cart, starting from $x_{1}(0)=0.5 \mathrm{~m}$ and $x_{2}(0)=0 \frac{\mathrm{m}}{\mathrm{s}}$. Figure 3(c) shows the control sequence. Figure 3(d) show the time evolution of $d_{1 \eta}$ and $d_{2}$. For $t \geq 1.6 s$ the signal $d_{2}$ is equal to zero. Note that, $\mathcal{H}_{\infty}$ and dual mode strategies guarantee ISS: cart position goes to zero when the disturbance vanishes. On the other hand, standard min-max strategy only guarantees ISpS. In fact, when the disturbance vanishes, cart position does not tend to the origin but to $x_{1}=0.1541 \mathrm{~m}$. Moreover, note that that $\mathcal{H}_{\infty}$ and dual mode performances are comparable since, in the neighborhood of the origin, the auxiliary control law is a good approximation of the $\mathcal{H}_{\infty}$ strategy.

\section{Conclusions}

In this paper a unified framework for the synthesis of min-max MPC control algorithms has been provided. The ISpS or ISS property of such algorithms is analyzed with respect to a general class of disturbances that considers both state dependent and state independent disturbances. The algorithms based on a standard stage cost, on an $\mathcal{H}_{\infty}$ cost and on a dual mode approach are compared. The relevance of the adopted stage cost to achieve ISS clarifies the difference between some of the results appeared in the literature.

\section{Appendix}

Definition 6 (Regional ISS in $\Xi$ with respect to $\mathcal{A}$ ) Suppose that Assumption 2-3 are satisfied. Given a closed set $\Xi \subset \mathcal{R}^{n}$ including the origin as an interior point, the system (7) with $\mathbf{d}_{\mathbf{1}} \in \mathcal{M}_{D_{1}}$ and $\mathbf{d}_{\mathbf{2}} \in \mathcal{M}_{D_{2}}$, is said to be ISS in $\Xi$ with respect to set $\mathcal{A}$ (compact-ISS), with respect to $d_{2}$, if $\Xi$ is robust positively invariant for (7) and if there exist a $\mathcal{K} \mathcal{L}$-function $\beta$ and a $\mathcal{K}$-function $\gamma_{2}$ such that

$$
\left|x\left(k, \bar{x}, \mathbf{d}_{\mathbf{1}}, \mathbf{d}_{\mathbf{2}}\right)\right|_{\mathcal{A}} \leq \beta\left(|\bar{x}|_{\mathcal{A}}, k\right)+\gamma_{2}\left(\left\|d_{2[k-1]}\right\|\right)
$$

for all $\bar{x} \in \Xi$ and $k>0$.

Definition 7 (LpS) Suppose that Assumption 2 is satisfied. System (7) with $\mathbf{d}_{\mathbf{1}} \in \mathcal{M}_{D_{1}}$ and $\mathbf{d}_{\mathbf{2}} \in \mathcal{M}_{D_{2}}$ satisfies the LpS (Local practical Stability) property if there exists a constant $c \geq 0$ such that for each $\varepsilon>0$, there exists a $\delta>0$ such that $\left|x\left(k, \bar{x}, \mathbf{d}_{\mathbf{1}}, \mathbf{d}_{\mathbf{2}}\right)\right| \leq c+\varepsilon, \forall k \geq 0$ for all $|\bar{x}| \leq \delta$ and all $\left|d_{2}(k)\right| \leq \delta$. 


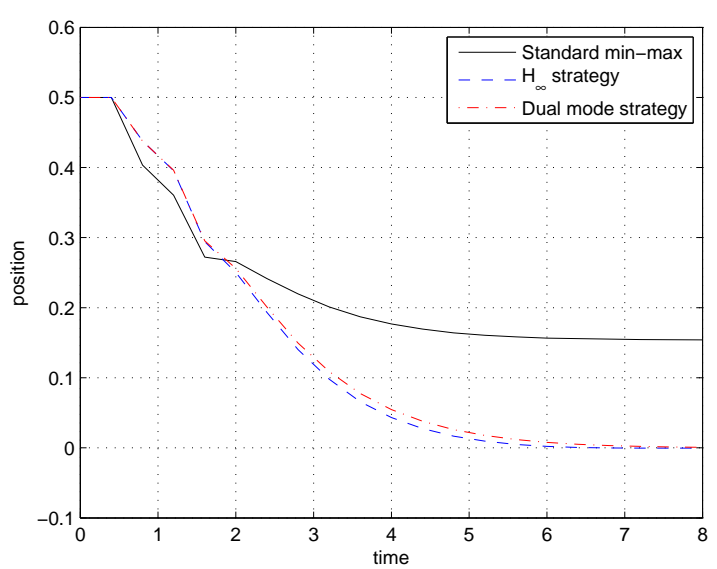

(a) Time evolution of cart position.

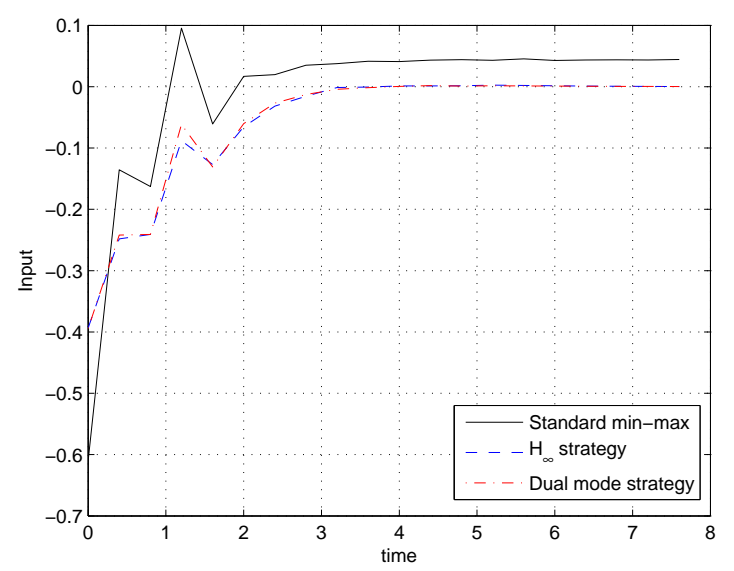

(b) Time evolution of cart velocity.

(c) Time evolution of the control.

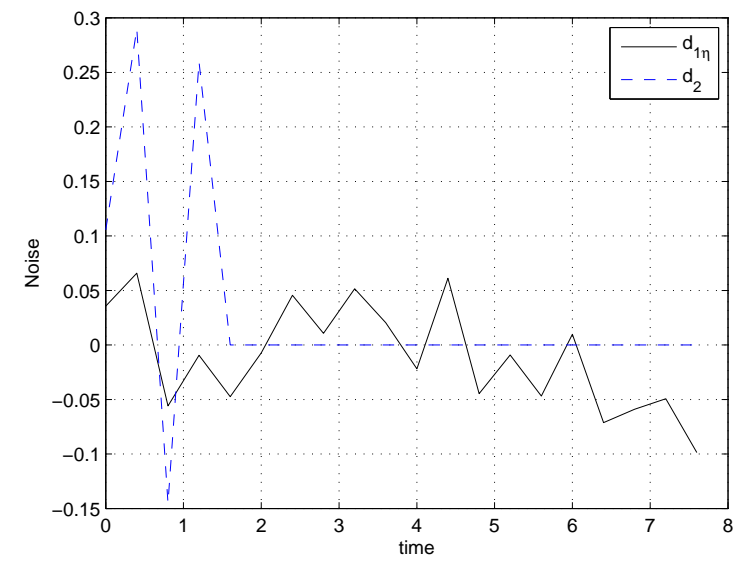

(d) Time evolution of uncertainty $d_{1 \eta}$ and disturbance $d_{2}$.

Figure 3: Simulation with initial conditions $x_{1}(0)=0.5 m$ and $x_{2}(0)=0 \frac{m}{s}$

Definition 8 (LS with respect to $\mathcal{A}$ ) Suppose that Assumption 2-3 are satisfied. System (7) with $\mathbf{d}_{\mathbf{1}} \in$ $\mathcal{M}_{D_{1}}$ and $\mathbf{d}_{\mathbf{2}} \in \mathcal{M}_{D_{2}}$ satisfies the LS (Local Stability) property with respect to set $\mathcal{A}$, if for each $\varepsilon>0$, there exists a $\delta>0$ such that $\left|x\left(k, \bar{x}, \mathbf{d}_{\mathbf{1}}, \mathbf{d}_{\mathbf{2}}\right)\right|_{\mathcal{A}} \leq \varepsilon, \forall k \geq 0$ for all $|\bar{x}|_{\mathcal{A}} \leq \delta$ and all $\left|d_{2}(k)\right| \leq \delta$.

Definition 9 (UpAG in $\Xi$ ) Suppose that Assumption 2 is satisfied. Given a closed set $\Xi \subseteq \mathcal{R}^{n}$ including the origin as an interior point, system (7) with $\mathbf{d}_{\mathbf{1}} \in \mathcal{M}_{D_{1}}$ and $\mathbf{d}_{\mathbf{2}} \in \mathcal{M}_{D_{2}}$ satisfies the UpAG (Uniform practical Asymptotic Gain) property in $\Xi$, if $\Xi$ is robust positively invariant for (7) and if there exists a constant $c \geq 0$ and $a \mathcal{K}$-function $\gamma_{2}$ such that for each $\varepsilon>0$ and $\nu>0, \exists T=T(\varepsilon, \nu)$ such that $\left|x\left(k, \bar{x}, \mathbf{d}_{\mathbf{1}}, \mathbf{d}_{\mathbf{2}}\right)\right| \leq \gamma_{2}\left(|| d_{2[k-1]}||\right)+c+\varepsilon$ for all $\bar{x} \in \Xi$ with $|\bar{x}| \leq \nu$, and all $k \geq T$. If $c=0$, the system is said to satisfy the UAG (Uniform Asymptotic gain) property.

Definition 10 (UAG in $\Xi$ with respect to $\mathcal{A}$ ) Suppose that Assumption 2-3 are satisfied. Given a closed set $\Xi \subseteq \mathcal{R}^{n}$ including the origin as an interior point, system (7) with $\mathbf{d}_{\mathbf{1}} \in \mathcal{M}_{D_{1}}$ and $\mathbf{d}_{\mathbf{2}} \in \mathcal{M}_{D_{2}}$ satisfies the UAG (Uniform Asymptotic Gain) property in $\Xi$, if $\Xi$ is robust positively invariant for (7) and if there exist a $\mathcal{K}$-function $\gamma_{2}$ such that for each $\varepsilon>0$ and $\nu>0, \exists T=T(\varepsilon, \nu)$ such that $\left|x\left(k, \bar{x}, \mathbf{d}_{\mathbf{1}}, \mathbf{d}_{\mathbf{2}}\right)\right|_{\mathcal{A}} \leq$ $\gamma_{2}\left(|| d_{2[k-1]}||\right)+\varepsilon$ for all $\bar{x} \in \Xi$ with $|\bar{x}|_{\mathcal{A}} \leq \nu$, and all $k \geq T$. 
Proof of Theorem 2: Let $\bar{x} \in \Xi$. The proof will be carried out in four steps.

Step 1: First we show that the set $\Theta$ defined in (12) is robust positively invariant for system (7). From the definition of $\bar{\alpha}_{2}(s)$ it follows that $\alpha_{2}(|x|)+c_{1} \leq \bar{\alpha}_{2}\left(|x|+c_{1}\right)$. Therefore $V(x) \leq \bar{\alpha}_{2}\left(|x|+c_{1}\right)$ and hence $|x|+c_{1} \geq \bar{\alpha}_{2}^{-1}(V(x)), \forall x \in \Omega$. Moreover (see [25]):

$$
\alpha_{3}(|x|)+\zeta\left(c_{1}\right) \geq \underline{\alpha}_{3}\left(|x|+c_{1}\right) \geq \alpha_{4}(V(x))
$$

where $\alpha_{4} \triangleq \underline{\alpha}_{3} \circ \bar{\alpha}_{2}^{-1}$ is a $\mathcal{K}_{\infty}$-function. Then

$$
\begin{aligned}
\Delta V(x) \leq & -\alpha_{4}(V(x))+\zeta\left(c_{1}\right)+c_{2}+\lambda\left(\left|d_{2}\right|\right) \\
= & -\alpha_{4}(V(x))+\lambda\left(\left|d_{2}\right|\right)+c_{3} \\
\leq & -\alpha_{4}(V(x))+\lambda\left(D_{2}^{\text {sup }}\right)+c_{3}, \\
& \forall x \in \Xi, \forall d_{1} \in D_{1}(x), \forall d_{2} \in D_{2}
\end{aligned}
$$

where $c_{3} \triangleq c_{2}+\zeta\left(c_{1}\right)$. Let us now assume that $x(k) \in \Theta$. Then $V(x(k)) \leq b\left(\lambda\left(D_{2}^{\text {sup }}\right)+c_{3}\right)$; this implies $\rho \circ \alpha_{4}(V(x)) \leq \lambda\left(D_{2}^{\text {sup }}\right)+c_{3}$. Without loss of generality, assume that $\left(i d-\alpha_{4}\right)$ is a $\mathcal{K}_{\infty}$-function (see Lemma B.1 [18]). Then

$$
\begin{aligned}
V\left(F\left(x, d_{1}, d_{2}\right)\right) \leq & \left(i d-\alpha_{4}\right)(V(x))+\lambda\left(D_{2}^{\text {sup }}\right)+c_{3} \\
\leq & \left(i d-\alpha_{4}\right)\left(b\left(\lambda\left(D_{2}^{\text {sup }}\right)+c_{3}\right)\right)+\lambda\left(D_{2}^{\text {sup }}\right)+c_{3} \\
= & -(i d-\rho) \circ \alpha_{4}\left(b\left(\lambda\left(D_{2}^{\text {sup }}\right)+c_{3}\right)\right)+b\left(\lambda\left(D_{2}^{\text {sup }}\right)+c_{3}\right) \\
& -\rho \circ \alpha_{4}\left(b\left(\lambda\left(D_{2}^{\text {sup }}\right)+c_{3}\right)\right)+\lambda\left(D_{2}^{\text {sup }}\right)+c_{3} .
\end{aligned}
$$

From the definition of $b$, it follows that $\rho \circ \alpha_{4}(b(s))=s$ and, owing to the fact that $(i d-\rho)$ is a $\mathcal{K}_{\infty}$-function, we obtain

$$
\begin{aligned}
V\left(F\left(x, d_{1}, d_{2}\right)\right) & \leq-(i d-\rho) \circ \alpha_{4}\left(b\left(\lambda\left(D_{2}^{\text {sup }}\right)+c_{3}\right)\right)+b\left(\lambda\left(D_{2}^{\text {sup }}\right)+c_{3}\right) \\
& \leq b\left(\lambda\left(D_{2}^{\text {sup }}\right)+c_{3}\right) .
\end{aligned}
$$

Hence $\Theta$ is robust positively invariant for system (7).

Step 2: Next, we show that the state, starting from $\Xi / \Theta$, tends asymptotically to $\Theta$. Firstly, if $x \in \Omega / \Theta$, then

$$
\rho \circ \alpha_{4}(V(x))>\lambda\left(D_{2}^{\text {sup }}\right)+c_{3} .
$$

From the inequality (19), we have

$$
\rho\left(\alpha_{3}(|x|)+\zeta\left(c_{1}\right)\right)>\lambda\left(D_{2}^{\text {sup }}\right)+c_{3} .
$$

On the other hand, $(i d-\rho)$ is a $\mathcal{K}_{\infty}$-function, hence

$$
i d(s)>\rho(s), \forall s>0
$$

then

$$
\alpha_{3}(|x|)+\zeta\left(c_{1}\right)>\rho\left(\alpha_{3}(|x|)+\zeta\left(c_{1}\right)\right)>\lambda\left(D_{2}^{\text {sup }}\right)+\zeta\left(c_{1}\right)+c_{2} .
$$

Hence

$$
\alpha_{3}(|x|)>\lambda\left(\left|d_{2}\right|\right)+c_{2}
$$

that means

$$
\Delta V(x)<0, \forall x \in \Omega / \Theta .
$$

Moreover, in view of (12), $\exists \bar{c}_{\theta}>0$ such that $\forall x_{1} \in \Xi / \Omega$, there exists $x_{2} \in \Omega / \Theta$ such that $\alpha_{3}\left(\left|x_{2}\right|\right) \leq$ $\alpha_{3}\left(\left|x_{1}\right|\right)-\bar{c}_{\theta}$. Then from (20) it follows that

$$
-\alpha_{3}\left(\left|x_{1}\right|\right)+\bar{c}_{\theta} \leq-\alpha_{3}\left(\left|x_{2}\right|\right)<-\lambda\left(\left|d_{2}\right|\right)-c_{2}, \forall x_{1} \in \Xi / \Omega .
$$


Then

$$
\Delta V(x)<-\bar{c}_{\theta}, \forall x \in \Xi / \Omega
$$

so that there exists $T_{1}$ such that

$$
x\left(T_{1}, \bar{x}, \mathbf{d}_{\mathbf{1}}, \mathbf{d}_{\mathbf{2}}\right) \in \Omega .
$$

Therefore, starting from $\Xi$, the state will reach the region $\Omega$ in a finite time. If $x\left(T_{1}, \bar{x}, \mathbf{d}_{\mathbf{1}}, \mathbf{d}_{\mathbf{2}}\right) \in \Theta$, the region $\Theta$ is achieved in a finite time. Since $\Theta$ is robust positively invariant, it is true that $\lim _{k \rightarrow \infty}\left|x\left(k, \bar{x}, \mathbf{d}_{\mathbf{1}}, \mathbf{d}_{\mathbf{2}}\right)\right|_{\Theta}=$ 0 . Otherwise, if $x\left(T_{1}, \bar{x}, \mathbf{d}_{\mathbf{1}}, \mathbf{d}_{\mathbf{2}}\right) \notin \Theta, \rho \circ \alpha_{4}\left(V\left(x\left(T_{1}, \bar{x}, \mathbf{d}_{\mathbf{1}}, \mathbf{d}_{\mathbf{2}}\right)\right)>\lambda\left(D_{2}^{\text {sup }}\right)+c_{3}\right.$ and

$$
\begin{aligned}
\Delta V\left(x\left(T_{1}, \bar{x}, \mathbf{d}_{\mathbf{1}}, \mathbf{d}_{\mathbf{2}}\right)\right) \leq & -\alpha_{4}\left(V\left(x\left(T_{1}, \bar{x}, \mathbf{d}_{\mathbf{1}}, \mathbf{d}_{\mathbf{2}}\right)\right)\right)+\lambda\left(D_{2}^{\text {sup }}\right)+c_{3} \\
= & -(i d-\rho) \circ \alpha_{4}\left(V\left(x\left(T_{1}, \bar{x}, \mathbf{d}_{\mathbf{1}}, \mathbf{d}_{\mathbf{2}}\right)\right)\right) \\
& -\rho \circ \alpha_{4}\left(V\left(x\left(T_{1}, \bar{x}, \mathbf{d}_{\mathbf{1}}, \mathbf{d}_{\mathbf{2}}\right)\right)\right)+\lambda\left(D_{2}^{\text {sup }}\right)+c_{3} \\
\leq & -(i d-\rho) \circ \alpha_{4}\left(V\left(x\left(T_{1}, \bar{x}, \mathbf{d}_{\mathbf{1}}, \mathbf{d}_{\mathbf{2}}\right)\right)\right) \\
\leq & -(i d-\rho) \circ \alpha_{4} \circ \alpha_{1}\left(\left|x\left(T_{1}, \bar{x}, \mathbf{d}_{\mathbf{1}}, \mathbf{d}_{\mathbf{2}}\right)\right|\right)
\end{aligned}
$$

where the last step is obtained using (9). Then, $\forall \varepsilon^{\prime}>0, \exists T_{2}\left(\varepsilon^{\prime}\right) \geq T_{1}$ such that

$$
V\left(x\left(T_{2}, \bar{x}, \mathbf{d}_{\mathbf{1}}, \mathbf{d}_{\mathbf{2}}\right)\right) \leq \varepsilon^{\prime}+b\left(\lambda\left(D_{2}^{\text {sup }}\right)+c_{3}\right) .
$$

Therefore, starting from $\Xi$, the state will arrive close to $\Theta$ in a finite time and to $\Theta$ asymptotically. Hence $\lim _{k \rightarrow \infty}\left|x\left(k, \bar{x}, \mathbf{d}_{\mathbf{1}}, \mathbf{d}_{\mathbf{2}}\right)\right|_{\Theta}=0$.

Step 3: Given $e \in \mathcal{R}_{\geq 0}$, let $R(e) \triangleq\{x: V(x) \leq e\}$. Let $\Psi \triangleq\left\{x: V(x) \leq \bar{e}=\max _{R(e) \subseteq \Omega} e\right\}$. It is clear that $\Psi \supseteq \Theta$ and that $\Psi$ is robust positively invariant. Since the upper bound of $V(x)$ is known in $\Psi \subseteq \Omega$ then, using the same steps of the proof of Lemma 3.5 in [18], the regional ISpS in $\Psi$ is obtained.

Step 4: Finally we show that system (7) is regional ISpS in $\Xi$. Using (21) and (9) there is

$$
\alpha_{1}\left(\left|x\left(T_{2}, \bar{x}, \mathbf{d}_{\mathbf{1}}, \mathbf{d}_{\mathbf{2}}\right)\right|\right) \leq V\left(x\left(T_{2}, \bar{x}, \mathbf{d}_{\mathbf{1}}, \mathbf{d}_{\mathbf{2}}\right)\right) \leq \varepsilon^{\prime}+b\left(\lambda\left(D_{2}^{\text {sup }}\right)+c_{2}\right)
$$

hence

$$
\left|x\left(T_{2}, \bar{x}, \mathbf{d}_{\mathbf{1}}, \mathbf{d}_{\mathbf{2}}\right)\right| \leq \alpha_{1}^{-1}\left(\varepsilon^{\prime}+b\left(\lambda\left(D_{2}^{\text {sup }}\right)+c_{2}\right)\right) .
$$

Noting that, given a $\mathcal{K}_{\infty}$-function $\theta_{1}, \theta_{1}\left(s_{1}+s_{2}\right) \leq \theta_{1}\left(2 s_{1}\right)+\theta_{1}\left(2 s_{2}\right)$, see [25], it follows that

$$
\left|x\left(T_{2}, \bar{x}, \mathbf{d}_{\mathbf{1}}, \mathbf{d}_{\mathbf{2}}\right)\right| \leq \alpha_{1}^{-1}\left(2 \varepsilon^{\prime}\right)+\alpha_{1}^{-1}\left(2 b\left(2 \lambda\left(D_{2}^{\text {sup }}\right)\right)\right)+\alpha_{1}^{-1}\left(2 b\left(2 c_{2}\right)\right) .
$$

Now, letting $\varepsilon \triangleq \alpha_{1}^{-1}\left(2 \varepsilon^{\prime}\right)$ and $\gamma\left(D_{2}^{\text {sup }}\right) \triangleq \alpha_{1}^{-1}\left(2 b\left(2 \lambda\left(D_{2}^{\text {sup }}\right)\right)\right)$ and $c \triangleq \alpha_{1}^{-1}\left(2 b\left(2 c_{2}\right)\right)$, the UpAG property in $\Xi$ is proven (if $c_{1}=0$ and $c_{2}=0$, the UAG property in $\Xi$ holds). In view of Theorem 1 , since the system is regional ISpS in $\Psi$, it is LpS and UpAG in $\Psi$. Finally, LpS with UpAG in $\Xi$ imply that the system is ISpS in $\Xi$ with respect to $d_{2}$. Moreover, if $c_{1}=0$ and $c_{2}=0$ the ISS in $\Xi$ holds.

Proof of Theorem 3: The robust positively invariance of $X^{M P C}(N)$ is easily derived from Assumption 5 by taking

$$
\bar{\kappa}_{t+1, t+N}=\left\{\begin{array}{cc}
\kappa_{t+1, t+N-1}^{o} & t+1 \leq k \leq t+N-1 \\
\kappa_{f}(x(t+N)) & k=t+N
\end{array}\right.
$$

as admissible policy vector at time $t+1$ starting from the optimal sequence $\kappa_{t, t+N-1}^{o}$ at time $t$. Moreover it is possible to show that $V(x, N)$ is an ISpS-Lyapunov function for the closed-loop system (1), (15). In fact

$$
\begin{aligned}
V(x, N) & =J\left(\bar{x}, \kappa_{t, t+N-1}^{o}, d_{1[t, t+N-1]}^{o}, d_{2[t, t+N-1]}^{o}, N\right) \geq \min _{\kappa_{t, t+N-1}} J\left(\bar{x}, \kappa_{t, t+N-1}, 0,0, N\right) \\
& \geq l\left(x, \kappa_{t, t}, 0,0\right) \geq \alpha_{l}(|x|), \forall x \in X^{M P C}(N) .
\end{aligned}
$$


In order to derive the upper bound, consider the following policy vector for the FHCLG with horizon $N+1$

$$
\tilde{\kappa}_{t, t+N}=\left\{\begin{array}{cc}
\kappa_{t, t+N-1}^{o} & t \leq k \leq t+N-1 \\
\kappa_{f}(x(t+N)) & k=t+N
\end{array}\right.
$$

Then

$$
\begin{aligned}
J\left(\bar{x}, \tilde{\kappa}_{t, t+N}, d_{1[t, t+N]}, d_{2[t, t+N]}, N+1\right)= & V_{f}(x(t+N+1))-V_{f}(x(t+N)) \\
& +l\left(x(t+N), u(t+N), d_{1}(t+N), d_{2}(t+N)\right) \\
& +\sum_{k=t}^{t+N-1} l\left(x(k), u(k), d_{1}(k), d_{2}(k)\right)+V_{f}(x(t+N))
\end{aligned}
$$

so that in view of Assumption 5

$$
J\left(\bar{x}, \tilde{\kappa}_{t, t+N}, d_{1[t, t+N]}, d_{2[t, t+N]}, N+1\right) \leq \sum_{k=t}^{t+N-1} l\left(x(k), u(k), d_{1}(k), d_{2}(k)\right)+V_{f}(x(t+N))+\varrho\left(\left|d_{2}\right|\right)
$$

which implies

$$
\begin{aligned}
& V(x, N+1) \leq \max _{d_{1 \eta} \in \mathcal{M}_{\mathcal{D}_{1 \eta}}, d_{2} \in \mathcal{M}_{\mathcal{D}_{2}}} J\left(\bar{x}, \tilde{\kappa}_{t, t+N}, d_{1[t, t+N]}, d_{2[t, t+N]}, N+1\right) \\
& \leq \max _{d_{1 \eta} \in \mathcal{M}_{\mathcal{D}_{1 \eta}}, d_{2} \in \mathcal{M}_{\mathcal{D}_{2}}} \sum_{k=t}^{t+N-1} l\left(x(k), u(k), d_{1}(k), d_{2}(k)\right)+V_{f}(x(t+N))+\varrho\left(\left|d_{2}\right|\right) \\
& =V(x, N)+\varrho\left(D_{2}^{\text {sup }}\right)
\end{aligned}
$$

which holds $\forall x \in X^{M P C}(N), \forall d_{1 \eta} \in \mathcal{M}_{\mathcal{D}_{1 \eta}}, \forall d_{2 \eta} \in \mathcal{M}_{\mathcal{D}_{2 \eta}}$

Moreover

$$
\begin{aligned}
V(x, N) & \leq V(x, N-1)+\varrho\left(D_{2}^{\text {sup }}\right) \leq \ldots \leq V(x, 0)+N \varrho\left(D_{2}^{\text {sup }}\right) \\
& =V_{f}(x)+N \varrho\left(D_{2}^{\text {sup }}\right)<\beta_{V_{f}}(|x|)+N \varrho\left(D_{2}^{\text {sup }}\right), \forall x \in X_{f} .
\end{aligned}
$$

From the monotonicity property (23) it is easily derived that

$$
\begin{aligned}
& V\left(f\left(x, \kappa^{M P C}(x), d_{1}, d_{2}\right), N\right)-V(x, N) \leq-l\left(x, \kappa^{M P C}(x), d_{1}, d_{2}\right)+\varrho\left(D_{2}^{\text {sup }}\right) \\
& \leq-\alpha_{l}(|x|)+\alpha_{d}\left(\left|d_{2}\right|\right)+\varrho\left(D_{2}^{\text {sup }}\right), \forall x \in X^{M P C}(N), \forall d_{1 \eta} \in \mathcal{D}_{1 \eta}, \forall d_{2} \in \mathcal{D}_{2} .
\end{aligned}
$$

Then, by (22), (24), (25), the ISpS with respect to $d_{2}$ is proven in $X^{M P C}(N)$.

Proof of Corollory 1: Proof of Corollary 1 is derived by proof of Theorem 3. One of the key steps in the proof of Theorem 3 is to show that condition 3) in Definition 3 is satisfied. In particular, using Assumption 4, point 5) of Assumption 5 and monotonicity property (23), it is shown that condition 3) in Definition 3 is satisfied by the inequality (25). Only ISpS can be proven because of term $\varrho\left(D_{2}^{s u p}\right)$ derived by term $\varrho\left(\left|d_{2}\right|\right)$ in point 5) of Assumption 5. The necessity of this term is related to the particular stage cost considered in the optimization problem. In Corallary 1 , a standard stage cost $l(x, u)$ is considered. In order to guarantee the satisfaction of Assumption 5 for a disturbance $d_{2}$ different from zero, term $\varrho\left(\left|d_{2}\right|\right)$ must be different from zero. However, note that in this case, by Assumption 7, $\alpha_{d} \equiv 0$. This fact, considering Assumption 6, leads to a less conservative estimation of the region $\Theta$ defined in (12).

Proof of Theorem 5: As shown in the proof of Theorem 3, Assumptions 1, 4-6 guarantee that the closedloop system (1)-(15) is ISpS in $X^{M P C}(N)$. Following the steps of the proof of Theorem 2, it can be proven that region $X_{f}$ is achieved in a finite time. The auxiliary control law is used when the state reaches the 
region $X_{f}$ or when it starts in $X_{f}$. By Assumption $5, V_{f}(x)$ is an ISS-Lyapunov function in $X_{f}$. Hence the closed-loop system with the auxiliary control law is ISS in $X_{f}$. ISS in $X_{f}$ is equivalent to UAG in $X_{f}$ and LS. Since $X_{f}$ is achieved in a finite time and system satisfies UAG property in $X_{f}$, UAG in $X^{M P C}(N)$ is obtained. Finally, by Theorem 1, the closed-loop system (1)-(16) is ISS in $X^{M P C}(N)$ since UAG in $X^{M P C}(N)$ and LS are equivalent to ISS in $X^{M P C}(N)$.

Proof of Proposition 1: Considering the particular auxiliary control law used, there exists a positive constant $r_{0}$ such that point 2) of Assumption 5 is satisfied for all $x$ belonging to

$$
\Omega_{0}=\left\{x:|x| \leq r_{0}\right\} \subset X .
$$

Define

$$
H(x, u, w)=V_{f}\left(f_{1}(x)+f_{2}(x) u+f_{3}(x) w\right)-V_{f}(x)+|z|^{2}-\gamma^{2}|w|^{2} .
$$

Then

$$
\begin{aligned}
H(x, u, w)= & \left(f_{1}(x)+f_{2}(x) u+f_{3}(x) w\right)^{\prime} P\left(f_{1}(x)+f_{2}(x) u+f_{3}(x) w\right) \\
& -x^{\prime} P x+h_{1}(x)^{\prime} h_{1}(x)+u^{\prime} u-\gamma^{2} w^{\prime} w \\
= & \left(f_{1}(x)^{\prime} P f_{1}(x)-x^{\prime} P x+h_{1}(x)^{\prime} h_{1}(x)\right)+u^{\prime}\left(f_{2}(x)^{\prime} P f_{2}(x)+I\right) u \\
& +w^{\prime}\left(f_{3}(x)^{\prime} P f_{3}(x)-\gamma^{2}\right) w+2\left[\begin{array}{ll}
u^{\prime} & w^{\prime}
\end{array}\right]\left[\begin{array}{l}
f_{2}(x)^{\prime} P f_{1}(x) \\
f_{3}(x)^{\prime} P f_{1}(x)
\end{array}\right] \\
& +2 u^{\prime} F_{2}(x)^{\prime} P F_{3}(x) w \\
= & \left(f_{1}(x)^{\prime} P f_{1}(x)-x^{\prime} P x+h_{1}(x)^{\prime} h_{1}(x)\right)+\left[\begin{array}{ll}
u^{\prime} & w^{\prime}
\end{array}\right] R(x)\left[\begin{array}{l}
u \\
w
\end{array}\right] \\
& +2\left[\begin{array}{ll}
u^{\prime} & w^{\prime}
\end{array}\right]\left[\begin{array}{c}
f_{2}(x)^{\prime} P f_{1}(x) \\
f_{3}(x)^{\prime} P f_{1}(x)
\end{array}\right]
\end{aligned}
$$

and, computing $H(x, u, w)$ for $u=\kappa^{*}(x)$ and $w=\xi^{*}(x)$,

$$
\begin{aligned}
& H\left(x, \kappa^{*}(x), \xi^{*}(x)\right)=\left(f_{1}(x)^{\prime} P f_{1}(x)-x^{\prime} P x+h_{1}(x)^{\prime} h_{1}(x)\right) \\
& +\left[\begin{array}{ll}
\kappa^{*}(x)^{\prime} & \xi^{*}(x)^{\prime}
\end{array}\right] R(x)\left[\begin{array}{l}
\kappa^{*}(x) \\
\xi^{*}(x)
\end{array}\right]-2\left[\begin{array}{ll}
\kappa^{*}(x)^{\prime} & \xi^{*}(x)^{\prime}
\end{array}\right] R(x)\left[\begin{array}{c}
\kappa^{*}(x) \\
\xi^{*}(x)
\end{array}\right] \\
& =\left(f_{1}(x)^{\prime} P f_{1}(x)-x^{\prime} P x+h_{1}(x)^{\prime} h_{1}(x)\right) \\
& -\left[f_{1}(x)^{\prime} P f_{2}(x) f_{1}(x)^{\prime} P f_{3}(x)\right] R(x)^{-1}\left[\begin{array}{l}
f_{2}(x)^{\prime} P f_{1}(x) \\
f_{3}(x)^{\prime} P f_{1}(x)
\end{array}\right]
\end{aligned}
$$

From (ii) it follows that there exist positive constants $\varepsilon, r$ such that

$$
H\left(x, \kappa^{*}(x), \xi^{*}(x)\right) \leq-\varepsilon|x|^{2}, \quad \forall x \in \Omega_{1}=\{x:|x| \leq r\} \subset \Omega_{0} .
$$

By the Taylor expansion Theorem (note that the first order term evaluated in $(u, w)=\left(\kappa^{*}(x), \xi^{*}(x)\right)$ and terms of order $>2$ are null)

$$
H(x, u, w)=H\left(x, \kappa^{*}(x), w^{*}\right)+\frac{1}{2}\left[\begin{array}{c}
u-\kappa^{*}(x) \\
w-\xi^{*}(x)
\end{array}\right]^{\prime} R(x)\left[\begin{array}{l}
u-\kappa^{*}(x) \\
w-\xi^{*}(x)
\end{array}\right]
$$

Assumption $(i)$ implies that there exists a neighborhood $X_{n e}$ of $x=0$ such that $\forall x \in X_{n e}, \quad r_{22}(x)<0$. If the system is controlled by $u=\kappa^{*}(x)$ then

$$
H\left(x, \kappa^{*}(x), w\right)=H\left(x, \kappa^{*}(x), \xi^{*}(x)\right)+\frac{1}{2}\left(w-\xi^{*}(x)\right)^{\prime} r_{22}(x)\left(w-\xi^{*}(x)\right) .
$$


Since $r_{22}(x)<0$, it follows that, given $X_{n e}$, there exist an open neighborhoods $W_{n e}$ of $w=0$ such that

$$
H\left(x, \kappa^{*}(x), w\right) \leq H\left(x, \kappa^{*}(x), \xi^{*}(x)\right), \forall x \in X_{n e}, \forall w \in W_{n e} .
$$

In view of (27), there exists a positive constant $r_{2}$ such that

$$
H\left(x, \kappa^{*}(x), w\right) \leq H\left(x, \kappa^{*}(x), \xi^{*}(x)\right) \leq-\varepsilon|x|^{2}
$$

$\forall x \in \Omega_{2}=\left\{x:|x| \leq r_{2}\right\} \subset \Omega_{1}$ and for all $w \in W_{n e}$. Let us choose some $\beta>0$ such that

$$
\Omega_{\beta}=\left\{x: V_{f}(x) \leq \beta\right\} \subset \Omega_{2} .
$$

From (26), (28), (29) follows that

$$
\begin{aligned}
V_{f}\left(f_{1}(x)+f_{2}(x) \kappa^{*}(x)+f_{3}(x) w\right) & \leq V_{f}(x)-|z|^{2}+\gamma^{2}|w|^{2}-\varepsilon|x|^{2} \\
& \leq V_{f}(x)-|z|^{2}+\gamma^{2}|w|^{2}, \forall x \in \Omega_{\beta}, \forall w \in W_{n e} .
\end{aligned}
$$

Hence, if $\mathcal{H}_{\infty}$ strategy is used with $l\left(x, u, d_{1}, d_{2}\right)=|z|^{2}-\gamma^{2}|w|^{2}$ and $\varrho \equiv 0$, point 5$)$ of Assumption 5 is satisfied. Consider now the case of $l\left(x, u, d_{1}, d_{2}\right)=\left|z_{l}\right|^{2}$ as defined in point $\left.b\right)$ of Proposition 1 (standard min-max stage cost)

$$
\begin{aligned}
V_{f}\left(f_{1}(x)+f_{2}(x) \kappa^{*}(x)+f_{3}(x) w\right) & \leq V_{f}(x)-|z|^{2}+\gamma^{2}|w|^{2} \\
& =V_{f}(x)-h_{1}(x)^{\prime} h_{1}(x)-u^{\prime} u+\gamma^{2} d_{1}^{\prime}(x) d_{1}(x)+\gamma^{2} d_{2}^{\prime} d_{2} .
\end{aligned}
$$

Using point (iii) of Proposition 1

$$
\begin{aligned}
V_{f}\left(f_{1}(x)+f_{2}(x) \kappa^{*}(x)+f_{3}(x) w\right) & \leq V_{f}(x)-h_{1}(x)^{\prime} h_{1}(x)-u^{\prime} u+\gamma^{2}\left(K_{d x}|x|^{2}+K_{d u}|u|^{2}\right)+\gamma^{2} d_{2}^{\prime} d_{2} \\
& \leq V_{f}(x)-h_{1}(x)^{\prime} h_{1}(x)-u^{\prime} u+a|x|^{2}+b|u|^{2}+\gamma^{2} d_{2}^{\prime} d_{2}
\end{aligned}
$$

with $a \triangleq \gamma^{2} K_{d x}$ and $b \triangleq \gamma^{2} K_{d u}$. Considering that point 2) of Assumption 5 is locally satisfied

$$
V_{f}\left(f_{1}(x)+f_{2}(x) \kappa^{*}(x)+f_{3}(x) w\right) \leq V_{f}(x)-h_{1}(x)^{\prime} h_{1}(x)-u^{\prime} u+a|x|^{2}+c|x|^{2}+\gamma^{2} d_{2}^{\prime} d_{2}
$$

with $c \triangleq b L_{f}^{2}$. Then, using point $b$ ) of Proposition 1

$$
\begin{aligned}
V_{f}\left(f_{1}(x)+f_{2}(x) \kappa^{*}(x)+f_{3}(x) w\right) & \leq V_{f}(x)-h_{l}(x)^{\prime} h_{l}(x)-u^{\prime} u+\gamma^{2}\left|d_{2}\right|^{2} \\
& \leq V_{f}(x)-h_{l}(x)^{\prime} h_{l}(x)-u^{\prime} u+\varrho\left(\left|d_{2}\right|\right) \\
& =V_{f}(x)-l(x, u)+\varrho\left(\left|d_{2}\right|\right), \forall x \in \Omega_{\beta}, \forall w \in W_{n e}
\end{aligned}
$$

where $\varrho(s) \triangleq \gamma^{2}|s|^{2}$. Hence point 5$)$ of Assumption 5 is satisfied for the standard min-max stage cost too.

In order to verify that Assumption 5 is satisfied, it remains to prove points 1),3), 4). Point 1) is obtained if $X_{f} \subseteq \Omega_{\beta} \subset X$. Point 4) is obviously satisfied since $V_{f}(x)=x^{\prime} P x$, with $P$ positive definite matrix, is such that

$$
\alpha_{V_{f}}(|x|) \triangleq \lambda_{\min }(P)\|x\|^{2} \leq x^{\prime} P x \leq \lambda_{\max }(P)\|x\|^{2} \triangleq \beta_{V_{f}}(|x|)
$$

where $\lambda_{\min }(P)$ and $\lambda_{\max }(P)$ are minimum and maximum eigenvalues of $P$.

In order to prove point 3), the robust invariance of $X_{f}$, let consider the proof of Theorem 2. By step 1, $\Theta\left(D_{2}^{\text {sup }}\right)$ is robust positively invariant. Since $\Theta\left(D_{2}^{\text {sup }}\right) \propto D_{2}^{\text {sup }}$, there exists a set $\bar{W}_{n e}=D_{1}(x) \times D_{2} \subset W_{n e}$ and a positive constant $\alpha$ such that, defining $X_{f}$ as

$$
X_{f} \triangleq\left\{x: V_{f}(x) \leq \alpha\right\}
$$

there is $\Theta \subset X_{f} \subset \Omega_{\beta}$. It is clear that $X_{f}$ is robust positively invariant.

The invariance of the closed-loop system (with the auxiliary control law) in $X_{f}$ ends the proof of Assumption 5 . 


\section{References}

[1] T. Alamo, D. Muñoz de la Peña, D. Limon, and E. F. Camacho. Constrained min-max predictive control: modifications of the objective function leading to polynomial complexity. IEEE Transactions on Automatic Control, 50(5):710-714, 2005.

[2] D. Angeli. An almost global notion of input-to-state stability. IEEE Transactions on Automatic Control, 49(6):866-874, 2004.

[3] E. F. Camacho and C. Bordons. Model Predictive Control. Springer, 2004.

[4] H. Chen, C. W. Scherer, and F. Allgöwer. A game theoretical approach to nonlinear robust receding horizon control of constrained systems. In American Control Conference '97, 1997.

[5] L. Chisci, A. Lombardi, and E. Mosca. Dual receding horizon control of constrained discrete-time systems. European Journal of Control, 2(4):278-285, 1996.

[6] D. DeHaan and M. Guay. Adaptive Robust MPC: A minimally-conservative approach. In American Control Conference 2007, pages 3937-3942, 2007.

[7] R. Findeisen, L. Imsland, F. Allgower, and B. A. Foss. State and output feedback nonlinear model predictive control: An overview. European Journal of Control, 9(2-3):190-206, 2003.

[8] F. A. C. C. Fontes and L. Magni. Min-max model predictive control of nonlinear systems using discontinuous feedbacks. IEEE Transactions on Automatic Control, 48(10):1750-1755, 2003.

[9] E. Franco, L. Magni, T. Parisini, M. Polycarpou, and D. Raimondo. Cooperative Constrained Control of Distributed Agents With Nonlinear Dynamics and Delayed Information Exchange: A Stabilizing Receding-Horizon Approach. IEEE Transactions on Automatic Control, 53(1):324-338, 2008.

[10] K. Gao and Y. Lin. On equivalent notions of input-to-state stability for nonlinear discrete time systems. In IASTED International Conference on Control and Applications, pages 81-86, 2000.

[11] P.J. Goulart, E.C. Kerrigan, and J.M. Maciejowski. Optimization over state feedback policies for robust control with constraints. Automatica, 42(4):523-533, 2006.

[12] G. Grimm, M. J. Messina, S. E. Tuna, and A. R. Teel. Examples when nonlinear model predictive control is nonrobust. Automatica, 40(10):1729-1738, 2004.

[13] G. Grimm, M. J. Messina, S. E. Tuna, and A. R. Teel. Nominally Robust Model Predictive Control With State Constraints. IEEE Transactions on Automatic Control, 52(10):1856-1870, 2007.

[14] L. Grune, E. D. Sontag, and F. R. Wirth. Asymptotic stability equals exponential stability, and ISS equals finite energy gain - if you twist your eyes. Systems $\&$ Control Letters, 38(2):127-134, 1999.

[15] Eva Gyurkovics. Receding horizon $\mathcal{H}_{\infty}$ control for nonlinear discrete-time systems. IEE Proceedings Control Theory and Applications, 149:540-546, 2002.

[16] Eva Gyurkovics and Tibor Takacs. Quadratic stabilisation with $\mathcal{H}_{\infty}$-norm bound of non-linear discretetime uncertain systems with bounded control. Systems \& Control Letters, 50:277-289, 2003.

[17] Z.-P. Jiang, Y. Lin, and Y. Wang. Nonlinear small-gain theorems for discrete-time feedback systems and applications. Automatica, 40:2129-2136, 2004.

[18] Z.-P. Jiang and Y. Wang. Input-to-state stability for discrete-time nonlinear systems. Automatica, $37: 857-869,2001$. 
[19] E. C. Kerrigan and D. Q. Mayne. Optimal control of constrained, piecewise affine systems with bounded disturbances. In 41st IEEE CDC, pages 1552- 1557, 2002.

[20] E.C. Kerrigan and J. M. Maciejowski. Feedback min-max model predictive control using a single linear program: robust stability and the explicit solution. International Journal of Robust and Nonlinear Control, 14(4):395-413, 2004.

[21] J.S. Kim, T.W. Yoon, A. Jadbabaie, and C. De Persis. Input-to-state stable finite horizon MPC for neutrally stable linear discrete-time systems with input constraints. Systems $\&$ Control Letters, 55(4):293-303, 2006.

[22] M. Lazar. Model predictive control of hybrid systems: Stability and robustness. PhD thesis, Eindhoven University of Technology, The Netherlands, 2006.

[23] M. Lazar, D. Muñoz de la Peña, W. P. M. H. Heemels, and T. Alamo. On input-to-state stability of min-max nonlinear model predictive control. Systems \& Control Letters, 57:39-48, 2008.

[24] D. Limon, T. Alamo, and E. F. Camacho. Input-to-state stable MPC for constrained discrete-time nonlinear systems with bounded additive uncertainties. In 41st IEEE CDC, pages 4619-4624, 2002.

[25] D. Limon, T. Alamo, F. Salas, and E. F. Camacho. Input to state stability of min-max MPC controllers for nonlinear systems with bounded uncertainties. Automatica, 42:797-803, 2006.

[26] D. Limon, J. M. Bravo, T. Alamo, and E. F. Camacho. Robust MPC of constrained nonlinear systems based on interval arithmetic. IEE Proceedings-Control Theory and Applications, 152:325, 2005.

[27] J. Löfberg. Minimax approaches to robust model predictive control. PhD thesis, Department of Electrical Engineering, Linköping University, Sweden, 2003.

[28] J.M. Maciejowski. Predictive Control: With Constraints. Prentice Hall, 2002.

[29] L. Magni, G. De Nicolao, R. Scattolini, and F. Allgöwer. Robust model predictive control of nonlinear discrete-time systems. International Journal of Robust and Nonlinear Control, 13:229-246, 2003.

[30] L. Magni, H. Nijmeijer, and A. J. van der Schaft. A receding-horizon approach to the nonlinear $H_{\infty}$ control problem. Automatica, 37:429-435, 2001.

[31] L. Magni, D. M. Raimondo, and R. Scattolini. Regional input-to-state stability for nonlinear model predictive control. IEEE Transactions on Automatic Control, 51(9):1548-1553, 2006.

[32] L. Magni and R. Scattolini. Control design for nonlinear systems: Trading robustness and performance with the model predictive control approach. IEE Proceedings - Control Theory \& Application, pages 333-339, 2005.

[33] L. Magni and R. Scattolini. Robustness and robust design of MPC for nonlinear discrete-time systems. In R. Findeisen et al. (Eds.): Assessment and Future Directions, volume LNCIS 358, pages 239-254. Springer-Verlag Berlin Heidelberg, 2007.

[34] D. Q. Mayne. Control of constrained dynamic systems. European Journal of Control, 7:87-99, 2001.

[35] D. Q. Mayne, J. B. Rawlings, C. V. Rao, and P. O. M. Scokaert. Constrained model predictive control: Stability and optimality. Automatica, 36:789-814, 2000.

[36] P. Mhaskar, N. H. El-Farra, and P. D. Christofides. Robust hybrid predictive control of nonlinear systems. Automatica, 41(2):209-217, 2005. 
[37] H. Michalska and D. Q. Mayne. Robust receding horizon control of constrained nonlinear systems. IEEE Transactions on Automatic Control, 38:1623-1633, 1993.

[38] D. Nesic and DS Laila. A note on input-to-state stabilization for nonlinear sampled-datasystems. IEEE Transactions on Automatic Control, 47(7):1153-1158, 2002.

[39] S. J. Qin and T. A. Badgwell. A survey of industrial model predictive control technology. Control Engineering Practice, 11(7):733-764, 2003.

[40] D. M. Raimondo, T. Alamo, D. Limon, and E. F. Camacho. Towards the practical implementation of min-max nonlinear Model Predictive Control. In 46th IEEE CDC, pages 1257-1262, 2007.

[41] D. M. Raimondo and L. Magni. A robust model predictive control algorithm for nonlinear systems with low computational burden. In IFAC Workshop on Nonlinear Model Predictive Control for Fast Systems, Grenoble, France, 2006.

[42] D. M. Raimondo, L. Magni, and R. Scattolini. Decentralized MPC of nonlinear systems: An input-tostate stability approach. International Journal of Robust and Nonlinear Control, 17:1651-1667, 2007.

[43] S. V. Raković, A. R. Teel, D. Q. Mayne, and A. Astolfi. Simple robust control invariant tubes for some classes of nonlinear discrete time systems. In 45th IEEE CDC, pages $6397-6402,2006$.

[44] JA Rossiter. Model-Based Predictive Control: A Practical Approach. CRC Press, 2003.

[45] P. O. M. Scokaert and D. Q. Mayne. Min-max feedback model predictive control for constrained linear systems. IEEE Transactions on Automatic Control, 43:1136-1142, 1998.

[46] P. O. M. Scokaert, D. Q. Mayne, and J. B. Rawlings. Suboptimal model predictive control (feasibility implies stability). IEEE Transactions on Automatic Control, AC-44:648-654, 1999.

[47] E. D. Sontag and Y. Wang. On characterizations of the input-to-state stability property. System E Control Letters, 24:351-359, 1995.

[48] E. D. Sontag and Y. Wang. New characterizations of input-to state-stability. IEEE Transactions on Automatic Control, 41:1283-1294, 1996.

[49] E.D. Sontag and Y. Lin. Stabilization with respect to noncompact sets: Lyapunov characterizations and effect of bounded inputs. In In Nonlinear Control Systems Design 1992, IFAC Symposia Series, M. Fliess Ed., Pergamon Press, Oxford, 1993, pages 43-49, 1992.

[50] E.D. Sontag and Y. Wang. On characterizations of input-to-state stability with respect to compact sets. In IFAC Non-Linear Control Systems Design Symposium, (NOLCOS '95), pages 226-231, Tahoe City, CA, 1995.

[51] J. Tsinias. Input to state stability properties of nonlinear systems and applications to bounded feedback stabilization using saturation. ESAIM: COCV, 2:57-87, 1997. 